Article

\title{
Multi-Omic Profiling of Macrophages Treated with Phospholipids Containing Omega-3 and Omega-6 Fatty Acids Reveals Complex Immunomodulatory Adaptations at Protein, Lipid and Metabolic Levels
}

\author{
Tatiana Maurício ${ }^{1}$, Susana Aveiro ${ }^{1,2} \mathbb{D}$, Sofia Guedes ${ }^{1}$, Diana Lopes ${ }^{1,3}$, Tânia Melo ${ }^{1,3}$, Bruno M. Neves ${ }^{4}$ (D), \\ Rosário Domingues ${ }^{1,3} \mathbb{D}$ and Pedro Domingues ${ }^{1, * \mathbb{D}}$
}

\section{check for}

updates

Citation: Maurício, T.; Aveiro, S.;

Guedes, S.; Lopes, D.; Melo, T.;

Neves, B.M.; Domingues, R.;

Domingues, P. Multi-Omic Profiling

of Macrophages Treated with

Phospholipids Containing Omega-3

and Omega-6 Fatty Acids Reveals

Complex Immunomodulatory

Adaptations at Protein, Lipid and

Metabolic Levels. Int. J. Mol. Sci.

2022, 23, 2139. https://doi.org/

$10.3390 /$ ijms23042139

Academic Editors: Maria Letizia Manca, Amparo Nacher, Mohamad Allaw, Matteo Perra and Ines Castangia

Received: 17 January 2022

Accepted: 10 February 2022

Published: 15 February 2022

Publisher's Note: MDPI stays neutral with regard to jurisdictional claims in published maps and institutional affiliations.

Copyright: (C) 2022 by the authors. Licensee MDPI, Basel, Switzerland. This article is an open access article distributed under the terms and conditions of the Creative Commons Attribution (CC BY) license (https:// creativecommons.org/licenses/by/ $4.0 /)$.
1 Mass Spectrometry Centre, LAQV-REQUIMTE, Department of Chemistry, Santiago University Campus, University of Aveiro, 3810-193 Aveiro, Portugal; tatianascm97@ua.pt (T.M.); s.aveiro@ua.pt (S.A.); sguedes@ua.pt (S.G.); dianasalzedaslopes@ua.pt (D.L.); taniamelo@ua.pt (T.M.); mrd@ua.pt (R.D.)

2 GreenCoLab-Green Ocean Association, University of Algarve, 8005-139 Faro, Portugal

3 CESAM-Centre for Environmental and Marine Studies, Department of Chemistry, Santiago University Campus, University of Aveiro, 3810-193 Aveiro, Portugal

4 Department of Medical Sciences and Institute of Biomedicine-iBiMED, University of Aveiro, 3810-193 Aveiro, Portugal; bruno.neves@ua.pt

* Correspondence: p.domingues@ua.pt

\begin{abstract}
In recent years, several studies have demonstrated that polyunsaturated fatty acids have strong immunomodulatory properties, altering several functions of macrophages. In the present work, we sought to provide a multi-omic approach combining the analysis of the lipidome, the proteome, and the metabolome of RAW 264.7 macrophages supplemented with phospholipids containing omega-3 (PC 18:0/22:6; $\omega 3$-PC) or omega-6 (PC 18:0/20:4; $\omega 6-$ PC) fatty acids, alone and in the presence of lipopolysaccharide (LPS). Supplementation of macrophages with $\omega 3$ and $\omega 6$ phospholipids plus LPS produced a significant reprogramming of the proteome of macrophages and amplified the immune response; it also promoted the expression of anti-inflammatory proteins (e.g., pleckstrin). Supplementation with the $\omega 3$-PC and $\omega 6$-PC induced significant changes in the lipidome, with a marked increase in lipid species linked to the inflammatory response, attributed to several pro-inflammatory signalling pathways (e.g., LPCs) but also to the pro-resolving effect of inflammation (e.g., PIs). Finally, the metabolomic analysis demonstrated that supplementation with $\omega 3-\mathrm{PC}$ and $\omega 6-\mathrm{PC}$ induced the expression of several metabolites with a pronounced inflammatory and anti-inflammatory effect (e.g., succinate). Overall, our data show that supplementation of macrophages with $\omega 3$-PC and w6-PC effectively modulates the lipidome, proteome, and metabolome of these immune cells, affecting several metabolic pathways involved in the immune response that are triggered by inflammation.
\end{abstract}

Keywords: macrophage; omega-3 phospholipid; omega-6 phospholipid; LPS; proteomics; lipidomics; metabolomics

\section{Introduction}

Macrophages are specialised cells of the innate immune system and the mononuclear phagocyte system and are strongly involved in all stages of inflammation [1]. Macrophages display a wide range of functions, such as (1) recognition of pathogen-associated molecular patterns (PAMPs) or danger-associated molecular patterns (DAMPs) [2,3]; (2) antigen presentation; (3) phagocytosis of microorganisms, debris, and apoptotic cells; and (4) the secretion of several molecules, including a variety of cytokines, lipid mediators $[1,4]$ and the production of reactive oxygen species (ROS), and reactive nitrogen species (RSN), such as nitric oxide (NO), to eliminate phagocytosed harmful organisms [3]. Two major 
populations of macrophages with distinct characteristics and functions have been established, depending on the microenvironmental conditions that drive their polarisation: M1like macrophages, also known as classically activated or pro-inflammatory macrophages and M2-like macrophages, or alternatively activated macrophages with a predominant anti-inflammatory role [4-6]. It is known that a balance between M1-like and M2-like macrophages is crucial for the resolution of inflammation and to ensure homeostasis [7].

Dietary intake of polyunsaturated fatty acids (PUFAs), mainly omega-3 and omega-6 FAs, is thought to modulate the functions of the innate and adaptive immune system with an increased ratio of omega-6/omega-3 PUFAs associated with the development or aggravation of inflammatory conditions [8,9]. PUFAs have been demonstrated to impact the functions of epithelial cells, dendritic cells, macrophages, neutrophils, and $\mathrm{T}$ and B cells $[2,10]$. Regarding the effects on macrophages, they mainly depend on direct and indirect interferences with inflammation-related signalling pathways [11]. These dietary compounds have been the subjects of numerous studies aimed to evaluate their immunomodulatory potential, particularly in the context of attenuating inflammation in chronic inflammatory-related disorders (reviewed in [12]).

The omega-3 PUFAs family includes three main acids, $\alpha$-linolenic acid (ALA), docosahexaenoic acid (DHA), and eicosapentaenoic acid (EPA) [8]. Omega-3 FAs and their respective metabolites may down-regulate the secretion of pro-inflammatory cytokines by macrophages and impair the activation of the NLRP3 inflammasome as well as the production of reactive oxygen species, while increasing phagocytic activity [13-15]. Omega-3 FAs have also been demonstrated to promote the polarisation of macrophages into the M2-like anti-inflammatory phenotype [13,16]. These immunomodulatory effects have been attributed to the regulation of key intracellular signalling cascades, such as the NF- $\mathrm{KB}$ and PPAR- $\gamma$ pathways or the GPR-120-mediated ERK activation [17-20]. These data indicate that omega-3 FAs may be useful in repressing macrophage-induced tissue inflammation in many chronic inflammatory diseases.

Omega- 6 fatty acids include linoleic acid (LA), $\gamma$-linolenic acid (GLA), and most importantly, arachidonic acid (AA) $[8,21]$. These compounds are generally associated with proinflammatory action and are linked to several chronic inflammatory disorders [22], although it has recently been demonstrated that omega- 6 fatty acids may have an anti-inflammatory effect [23]. Similarly to omega-3 fatty acids, omega-6 FAs are also associated with multiple effects on immune cells, particularly on macrophages [8]. Supplementation with omega-6 FAs, in particular LA and AA, is classically described to enhance the macrophage production of inflammatory cytokines, such as IL-6 and TNF-a and the production of ROS, as well as their phagocytic capacity [24-27]. However, there is increasing evidence that omega- 6 fatty acids and their derivatives may also exert anti-inflammatory effects. For example, it has been demonstrated in vitro that LA promotes a decrease in macrophage production of IL- 6 and TNF- $\alpha$ while increasing the levels of the anti-inflammatory cytokine interleukin 10 [24]. Additionally, AA and LA can be metabolised to pro-resolving lipoxins and CYP450-derived oxylipins, and the omega-6 PUFAs dihomo- $\gamma$-linolenic acid (DGLA) and adrenic acid ( $\mathrm{AdA}$ ) could compete with $\mathrm{AA}$ for metabolisation, reducing the formation of pro-inflammatory mediators [28]. Recently, AdA has been demonstrated to block in vivo the production of LTB4 by neutrophils and to enhance macrophage efferocytosis of apoptotic neutrophils in an in vivo murine model of arthritis [29]. Finally, several meta-analysis studies report the beneficial effects of dietary intake of omega-6 PUFAs, such as LA [30,31].

Therefore, the dichotomous classification of omega-3 and omega-6 PUFAs as being exclusively anti- and pro-inflammatory, respectively, is too simplistic, indicating that further studies with broader and integrative approaches are needed to shed light on the effects of these bioactive lipids. For example, studies simultaneously describing the impact of omega3 and omega- 6 fatty acids on lipids, proteins, and metabolites remodelling in macrophages are still scarce.

Thus, with this study, we sought to provide a multi-omic analysis, involving lipidomics, proteomics, and metabolomics approaches based on a liquid chromatography-mass spec- 
trometry (LC-MS) analysis of cell extracts from the RAW 264.7 macrophage-like cell line exposed to phospholipids containing omega- 3 and omega- 6 fatty acids. The effects of FAs were studied on resting macrophages and M1-like pro-inflammatory macrophages polarised by exposure to LPS.

\section{Results}

2.1. Impact of Omega-3 and Omega-6 PCs on Macrophage Viability and LPS-Triggered Nitric Oxide Production

We first determined the working concentration of omega-3 ( $\omega 3-\mathrm{PC})$ and omega- 6 ( $\omega 6-\mathrm{PC})$ phospholipids by evaluating their toxicity, (Alamar Blue reduction assay), as well as the anti-inflammatory activity of the compounds (Griess Reaction assay). The results in Figure 1a show that the number of viable cells remained high for concentrations up to $100 \mu \mathrm{M}$. Only treatment with $200 \mu \mathrm{M}$ for both $\omega 3$-PC and w6-PC phospholipids resulted in a significant decrease in cell viability compared with control cells.

a)

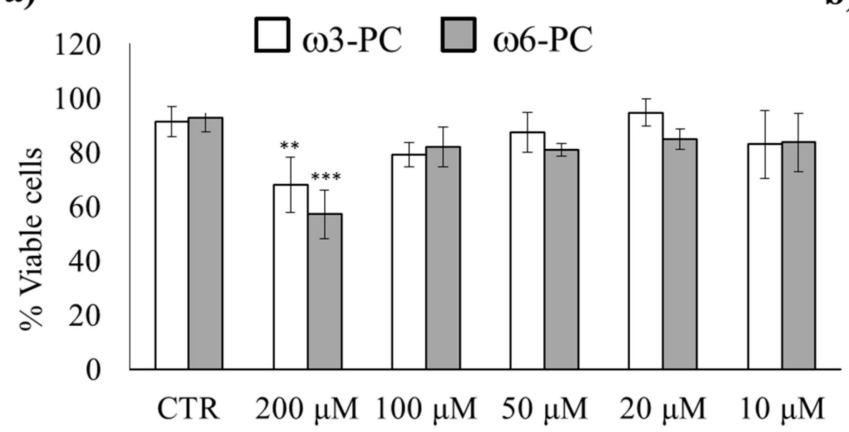

b)

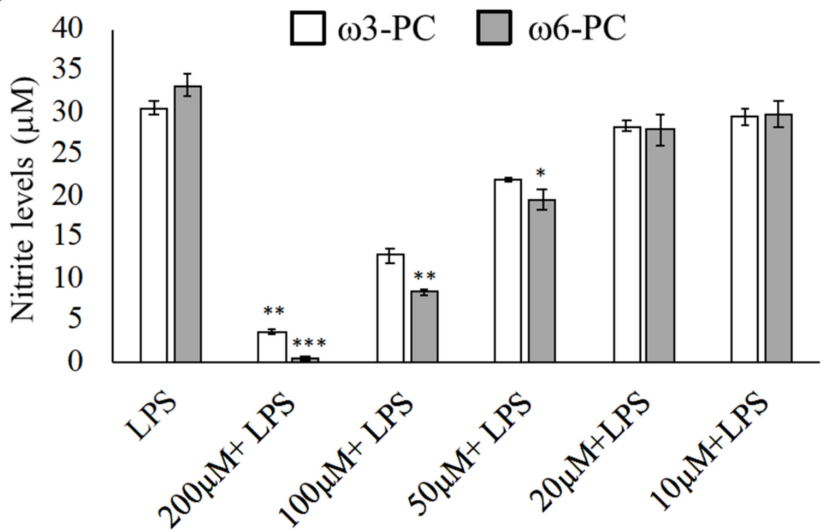

Figure 1. Impact of $\omega 3-\mathrm{PC}$ and $\omega 6-\mathrm{PC}$ on macrophage viability and LPS-triggered nitric oxide production (a). Raw 264.7 macrophages were incubated with 10-200 $\mu \mathrm{M}$ of $\omega 3$-PC and $\omega 6-\mathrm{PC}$ for $24 \mathrm{~h}$. $(n=4)$ and cell viability was assessed by the resazurin reduction assay $(\mathbf{b})$. Raw 264.7 macrophages were incubated with 0-200 $\mu \mathrm{M}$ of $\omega 3$-PC and $\omega 6-P C$ plus LPS for $24 \mathrm{~h}$ and the levels of nitrites in the culture medium were then determined by performing the Griess assay $(n=3) .{ }^{*} p<0.05,{ }^{* *} p<0.01$, $* * * p<0.001$, compared with the control group (CTRL). Error bars represented as mean $\pm \mathrm{SD}$.

The effect of the phospholipids on LPS-induced NO production was then investigated. NO production was suppressed upon treatment with $\omega 3-\mathrm{PC}$ and $\omega 6-\mathrm{PC}$ in a dose-dependent manner (Figure 1b). A significant decrease in NO production was observed with 100 and $200 \mu \mathrm{M}$ of $\omega 3-\mathrm{PC}$ and with 50, 100, and $200 \mu \mathrm{M}$ of $\omega 6-\mathrm{PC}$. Therefore, to prevent the possible influence of unspecific mechanisms triggered by cytotoxicity, we used the subtoxic concentration of $100 \mu \mathrm{M}$ for both w3-PC and w6-PC in the following experimental procedures.

\subsection{Supplementation with Omega-3 and Omega-6 PCs Promotes Macrophage Proteome Noticeable Remodelling}

In the proteomic analysis, we identified and semi-quantified a total of 3238 proteins (Supplementary Table S1), of which 228 were involved in lipid metabolism and 461 were involved in the regulation of the immune system (https://www.ebi.ac.uk/QuickGO/ (accessed on 15 June 2021)). The PCA score plot (Figure 2), shows that the six groups studied were well clustered according to changes in the proteomic profile and all samples were well separated by the first two dimensions (Dim1 52.6\% and Dim2 8.5\%). 


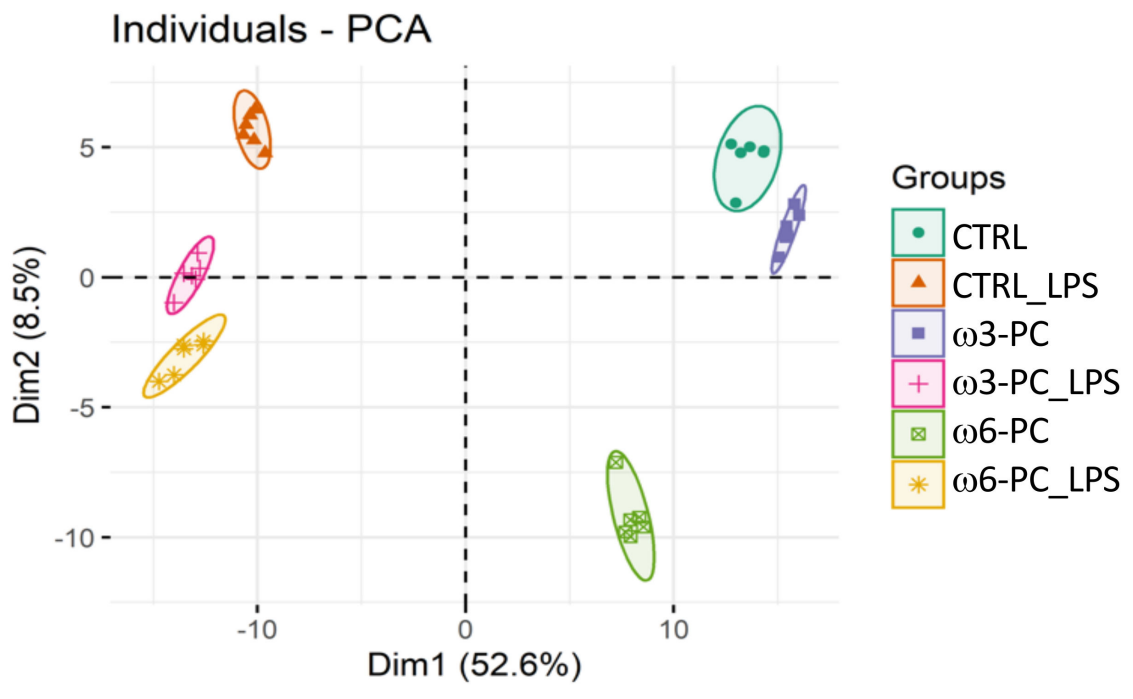

Figure 2. Principal component analysis (PCA) score plot of the proteomic profile, obtained for macrophages incubated with omega-3 and omega- 6 phospholipids ( $\omega 3-\mathrm{PC}$ and $\omega 6-\mathrm{PC})$, with omega3 and omega-6 phospholipids and LPS (w3-PC_LPS and w6-PC_LPS) and controls (CTRL and CTRL_LPS) $(n=6)$.

Comparing the relative abundance of the proteins identified in macrophages control samples (CTRL) and samples treated with omega-3 ( $\omega 3-\mathrm{PC})$ or omega-6 ( $\omega 6-\mathrm{PC})$ phospholipids, the number of significantly different proteins was 225 and 726, respectively (Table 1). Comparing macrophages samples treated with $\omega 3-\mathrm{PC}$ and $\omega 6-\mathrm{PC}$ revealed that the relative abundance of 887 proteins was significantly different. For the samples treated with LPS, 1854 proteins changed significantly between $\omega 3$ and $\omega 3$-PC_LPS, and 1256 proteins between $\omega 6$-PC and $\omega 6$-PC_LPS. Between the $\omega 3$-PC_LPS group and the $\omega 6$-PC_LPS group, only 526 proteins were significantly different.

Table 1. Number of proteins with significantly different relative abundance $(\mathrm{q}<0.05)$ between conditions (FDR adjusted Dunn's multiple comparison test).

\begin{tabular}{cccccc}
\hline & CTRL & $\omega 3-P C$ & $\omega 6-P C$ & CTRL_LPS & $\omega 3-P C \_L P S$ \\
\hline CTRL & - & & & & \\
$\omega 3-P C$ & 215 & - & - & & \\
$\omega 6-P C$ & 726 & 887 & 993 & - & \\
CTRL_LPS & 1346 & 2173 & 1182 & 480 & - \\
$\omega 3$-PC_LPS & 1498 & 1854 & 1256 & 225 & 526 \\
$\omega 6$-PC_LPS & 2008 & 2541 & & & \\
\hline
\end{tabular}

The clustering and functions of the 50 most significantly modulated proteins can be visualised in a two-dimensional hierarchical clustering heatmap, as shown in in Figure 3, Supplementary Table S2, in the Supplementary Materials. The dendrogram at the top of the heatmap shows that the samples have been clustered in the first leaf into two groups and are clearly dependent on LPS treatment. The first group (on the left) contains all experimental conditions exposed to LPS and the second group (on the right) includes conditions in which macrophages were not treated with the bacterial PAMP. The dendrogram on the right side of the heatmap shows the proteins annotated according to GO functions. In the first leaf, the proteins were clustered into two groups; the first group contained 13 proteins, seven of which (P14901, Q64337, Q8R2Q8, P11928, Q60766, Q9JHK5, and P01900) are involved in the immune system and are upregulated in response to LPS treatment. It should be noted that the increase triggered by LPS observed in the 13 proteins of this first cluster is higher when the cells were simultaneously exposed to PC, the $\omega 3$ phospholipid being the one that caused the most pronounced effects. Exposure to PCs alone caused minimal changes 
in these proteins. The second group contained 37 proteins, which are mainly involved in lipid metabolic processes and response to a stimulus. The proteins clustering in this group are overall downregulated by LPS treatment independently of the presence of PCs, and strongly upregulated by culture supplementation with the $\omega 3$ phospholipid. In this second leaf, a sub-group of 13 proteins (O88569, P61164, Q60737, Q9CZ13, P70404, P97807.1, P56480, P05202, Q9DB77, Q8QZT1, Q8BFR5, and Q9D6R2) is strongly downregulated by $\omega 6$ PC either in the presence or absence of LPS. Among the 37 proteins, five (P41241, P00493, Q00612, Q9ESY9, and Q09014) are involved in the immune system. Another sub-group of proteins can be noted in the second leaf of the dendrogram, consisting of only four proteins (Q6ZQM8.1 (lipid metabolic process), P24270 (lipid metabolic process), O09172 (response to stimulus), and Q923D2 (metabolic process)), the expression of which was markedly increased by both $\omega 3$ and $\omega 6$ phospholipids.

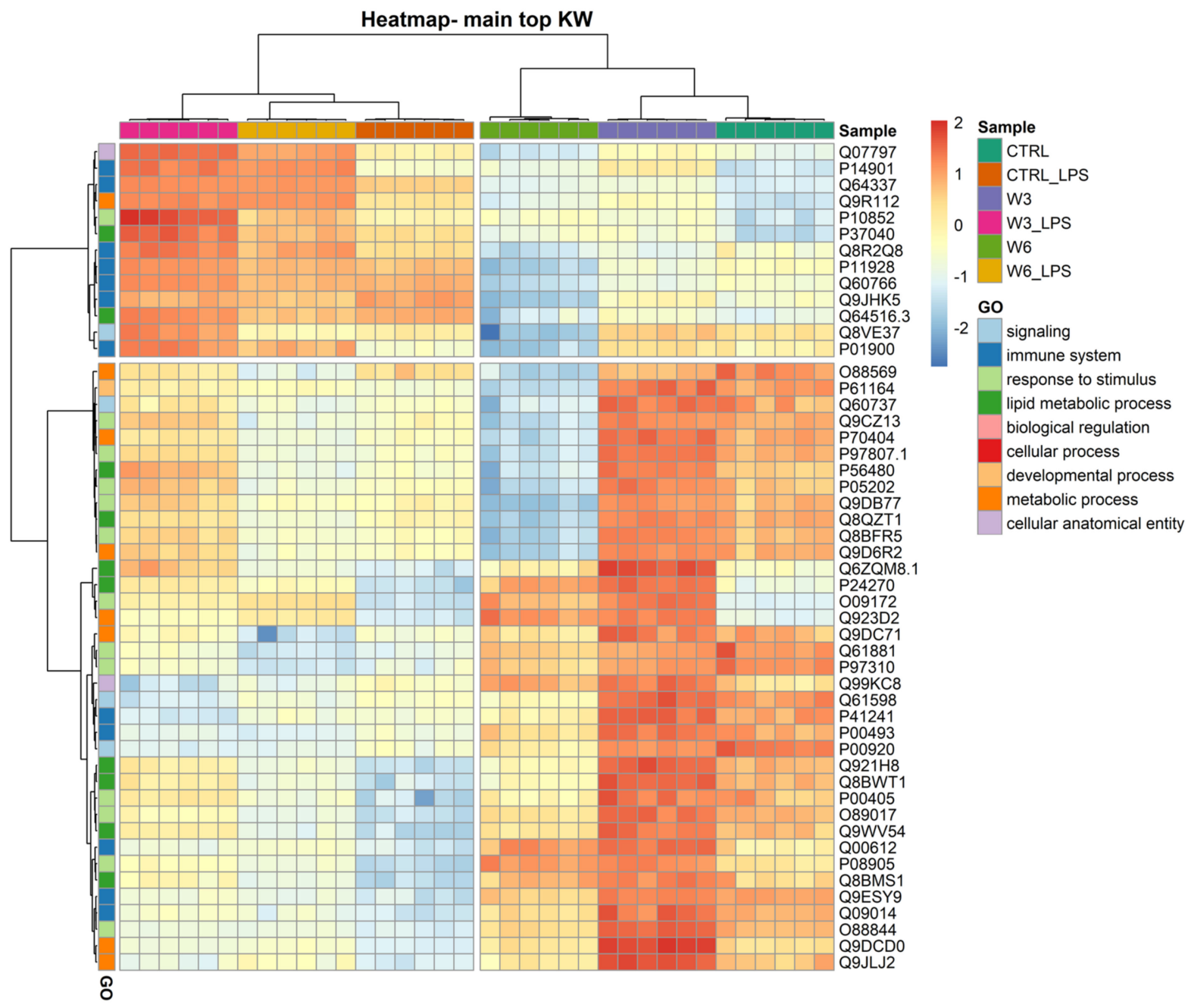

Figure 3. Two-dimensional hierarchical clustering heatmap of the 50 main proteins (lower q values) under the 6 conditions: control (CTRL), control for LPS (CTRL_LPS), omega-3 PC (w3-PC), omega-3

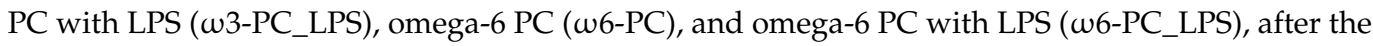
Kruskal-Wallis test. At the top is the dendrogram of the samples, and on the left is the dendrogram of proteins labelled with their generic function according to GO analysis.

\subsection{Lipidomics}

To characterise the lipid profile of macrophages, identification and quantification of lipid species were performed using high-resolution HILIC-MS/MS. In the lipidomic analysis, we identified and semi-quantified a total of 299 individual polar lipid species, including glycerophospholipids, glycerolipids, sphingolipids, and acyl-carnitines. In total, 
13 classes of lipid species have been identified: acyl-carnitines (CAR), di-acylglycerols (DG), ceramides (Cer), hexosylceramides (HexCer), sphingomyelins (SM), phosphatidylcholines (PC), phosphatidylethanolamines (PE), phosphatidylinositols (PI), phosphatidylglycerols (PG), phosphatidylserine (PS), cardiolipins (CL), lysophosphatidylcholine (LPC), and lysophosphatidylethanolamines (LPE). Some classes, such as PC, PE, LPC, and LPE, also include alkylacyl lipids. DG, CAR, Cer, HexCer, SM, LPC, and PC species were identified in the positive ion mode, as $[\mathrm{M}+\mathrm{H}]^{+}$ions. In the negative mode, $\mathrm{LPE}, \mathrm{PE}, \mathrm{PI}, \mathrm{PG}$, and PS were identified as $[\mathrm{M}-\mathrm{H}]^{-}$ions and CLs as $[\mathrm{M}-2 \mathrm{H}]^{2-}$ molecular ions. All identified lipid species can be found in Supplementary Table S3.

The PCA analysis of the LC-MS dataset shows the clustering of samples into the six groups (CTRL, CTRL_LPS, w3-PC, w3-PC_LPS, w6-PC, and w6-PC_LPS), which were well separated with no outliers (Figure 4). A clear distinction can also be observed between groups treated with (CTRL_LPS, w3-PC_LPS, and w6-PC_LPS) or without (CTRL, w3-PC, and w6-PC) LPS, being well separated along Dim1 (34.2\%).

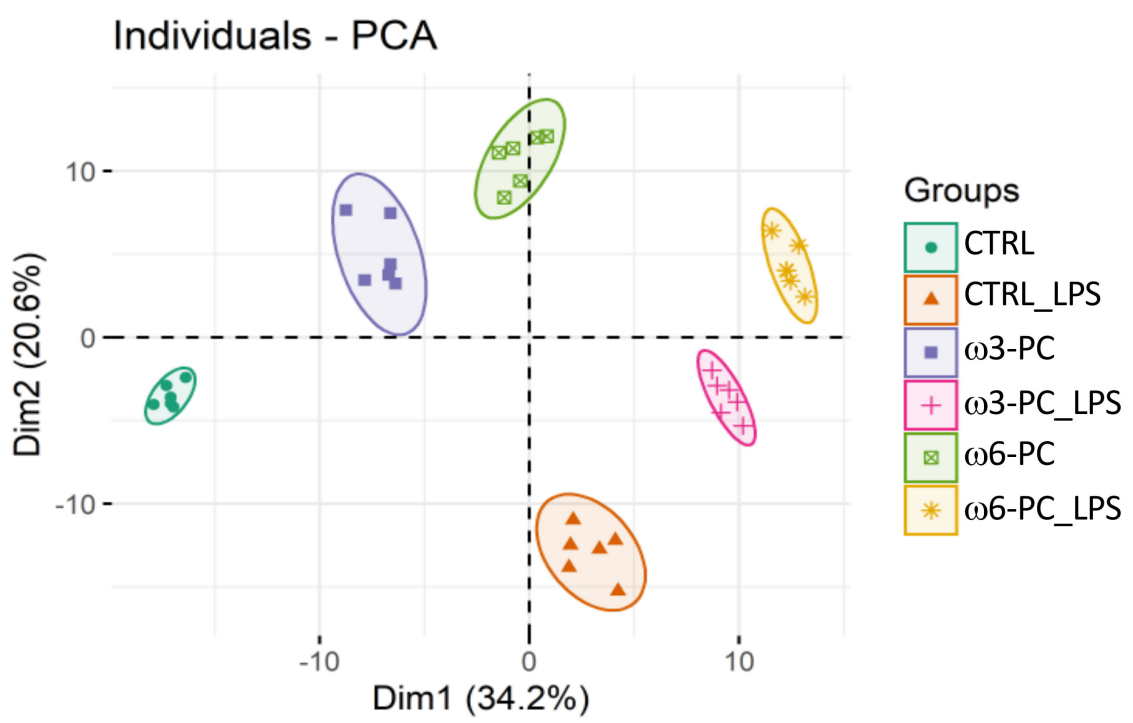

Figure 4. Principal component analysis (PCA) score plot of the phospholipid dataset acquired by LC-MS analysis, for the 6 groups: control (CTRL), control with LPS treatment (CTRL_LPS), $\omega 3-P C$,

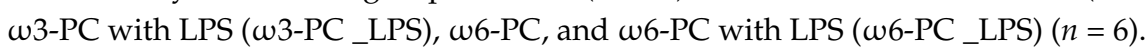

There were 273 lipid species, whose relative abundance changed significantly between conditions ( $q$-value $<0.05$ ) (Supplementary Table S4). The relative abundance of several species belonging to different classes, was significantly altered between conditions, with a $q$-value $<0.05$ and a fold change $>1.5$, such as in the Cer, CL, DG, LPC, LPE, PC, PG, and PI species (Table 2). Little to no variation was observed when comparing the control group vs. macrophages cultured with PCs (CTRL vs. $\omega 3$-PC and CTRL vs. w6-PC), although $\omega-6$ PC promoted lipidome remodelling to a greater extent compared with the $\omega-3$ phospholipid (three vs. 14 species altered). This stronger effect of $\omega 6$-PC on the macrophage lipidome was also observed when comparing cells treated with LPS (CTRL-LPS) with $\omega 3$-LPS or $\omega 6-$ LPS. In macrophages activated by LPS, the culture supplementation with w6-PC caused significant alterations in 23 lipid species, whereas supplementation with $\omega 3$-PC caused differences in only five species (Table 2). When comparing untreated cells with those exposed to LPS (CTRL vs. CTRL_LPS), 44 lipids were significantly modulated, mainly including the upregulation of Cer, CL, DG, PI, LPE, and LPC. 
Table 2. Number of lipids with fold change $(\mathrm{FC})>1.5$ between conditions and $\mathrm{q}<0.05$. Control

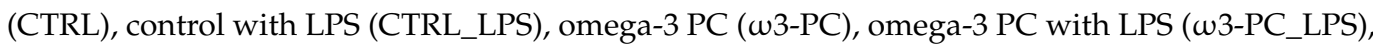

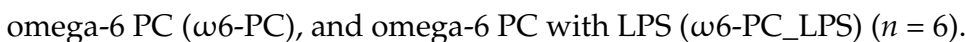

\begin{tabular}{|c|c|c|c|c|c|}
\hline & CTRL & $\omega 3-P C$ & $\omega 6-P C$ & CTRL_LPS & $\omega 3-P C \_L P S$ \\
\hline$\omega 3$ & 3 (1CL; 1LPC; 1PC) & - & & & \\
\hline$\omega 6$ & $\begin{array}{c}14 \text { (2CAR; 5DG; 3LPE; } \\
\text { 2PE; } 2 \text { PI) }\end{array}$ & 0 & - & & \\
\hline CTRL-LPS & $\begin{array}{c}44 \text { (2CER;8CL; 10DG;1 } \\
\text { HEXCER; 9LPE; 1PE; } \\
\text { 5PG; 8PI) }\end{array}$ & $\begin{array}{l}25 \text { (1CAR;1CER;5CL; } \\
\text { 7DG; 4LPE; 3PC; } \\
\text { 3PG; 1PI) }\end{array}$ & $\begin{array}{l}19 \text { (3CAR;2CER;3CL; } \\
\text { 1DG;5PC;1PE; } \\
\text { 3PG; 1PI) }\end{array}$ & - & \\
\hline$\omega 3-P C \_L P S$ & $\begin{array}{c}53 \text { (5CER;5CL; } \\
\text { 7DG;7LPC;9LPE; 2PC; 3PG; } \\
\text { 12PI; 2SM) }\end{array}$ & $\begin{array}{l}34 \text { (2CAR; 2CER; 2CL; } \\
\text { 2DG;7LPC;4LPE; 1PC; } \\
\text { 2PG; 11PI) }\end{array}$ & $\begin{array}{l}16 \text { (2CAR;3CER;2CL; } \\
\text { 2LPC;1PE; 2PG; 4PI) }\end{array}$ & 5 (1DG;3LPC;1PC) & - \\
\hline$\omega 6-P C \_L P S$ & $\begin{array}{l}\text { 66 (1CAR;4CER;5CL; } \\
\text { 8DG;11LPC; 14LPE; 4PC; } \\
\text { 1PE; 4PG; 12PI; 1SM) }\end{array}$ & $\begin{array}{c}49 \text { (2CER;4CL; } \\
\text { 6DG;10LPC; 9LPE; 2PC; } \\
\text { 1PE; 2PG; 12PI; 1SM) }\end{array}$ & $\begin{array}{c}31 \text { (1CAR; } \\
\text { 2CER;3CL;5LPC;3LPE; } \\
\text { 3PC; 4PG; 9PI; 1SM) }\end{array}$ & $\begin{array}{l}23 \text { (1CAR; 1CL; 3DG; } \\
\text { 8LPC;2LP; 6PC;2SM) }\end{array}$ & 3 (2LPE; 1PC) \\
\hline
\end{tabular}

\subsection{Metabolomics}

LC-MS based untargeted metabolomics provides in-depth profiling of the macrophage metabolome, revealing changes in metabolite abundance upon exogenous stimuli. Our analysis allowed us to identify and quantify a total of 103 species of metabolites, detailed in Supplementary Table S5.

The PCA analysis showed the clustering of conditions into three main groups: macrophages untreated or cultured with PC (CTRL; $\omega 3-P C ; \omega 6-P C)$, macrophages treated with LPS (CTRL_LPS), and cells cultured with PC and treated with LPS ( $\omega 3$-PC_LPS, w6-PC_LPS) (Figure 5). As observed for the impact on the lipidome, PC has little effect on the metabolome of macrophage, with w6-PC again having a more pronounced effect than w3-PC. The activation of macrophages with LPS had a considerable impact on the metabolome compared to untreated cells (main discriminant component Dim1, 35.2\%) and the concomitant culture with PCs notably modifies these alterations triggered by LPS.

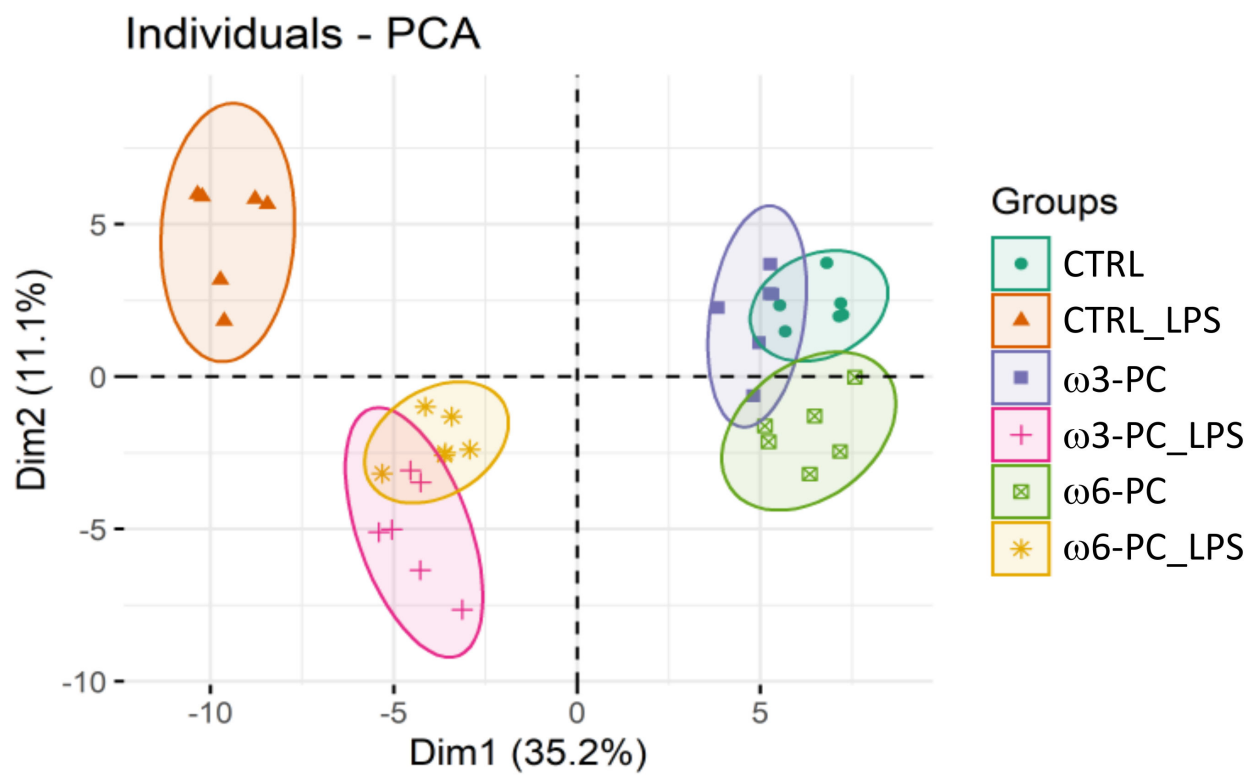

Figure 5. Principal component analysis (PCA) score plot of the metabolite species dataset acquired by LC-MS analysis for the 6 groups: control (CTRL), control for LPS (CTRL_LPS), w3-PC, w3-PC

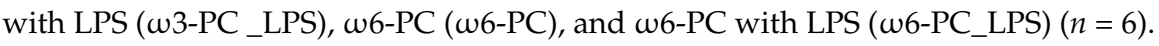


Differential analysis of cellular metabolites in the comparison of CTRL and CTRL-LPS was associated with 33 metabolic pathways (FDR $<0.05)$ including: histidine metabolism ( $p$-value $<0.001$, FDR $<0.001)$; alanine, aspartate, and glutamate metabolism ( $p$-value $<0.001$, FDR < 0.001); D-glutamine and D-glutamate metabolism ( $p$-value $<0.001$, FDR $<0.001)$; glutathione metabolism ( $p$-value $<0.001$, FDR $<0.001)$; and cysteine and methionine metabolism ( $p$-value $<0.001$, FDR $<0.001)$, (Figure 6 and Supplementary Table S6). The boxplots in Figure 6A,B show a general trend for the decrease of the relative abundance of metabolites linked to these pathways when cells were stimulated with LPS (CTRL_LPS). Statistically significant differences were observed in the glutathione metabolic pathway, with the concentration of glycine and glutamate decreased upon treatment with LPS. However, no significant differences were found in the concentration of glutathione. The histidine and aspartate metabolic pathways were also significantly modulated, with LPS causing a significant increase in the levels of L-histidine and N-methyl-L-histidine, while decreasing L-glutamate and histamine.

\section{CTRL vs CTRL_LPS}

\section{A}

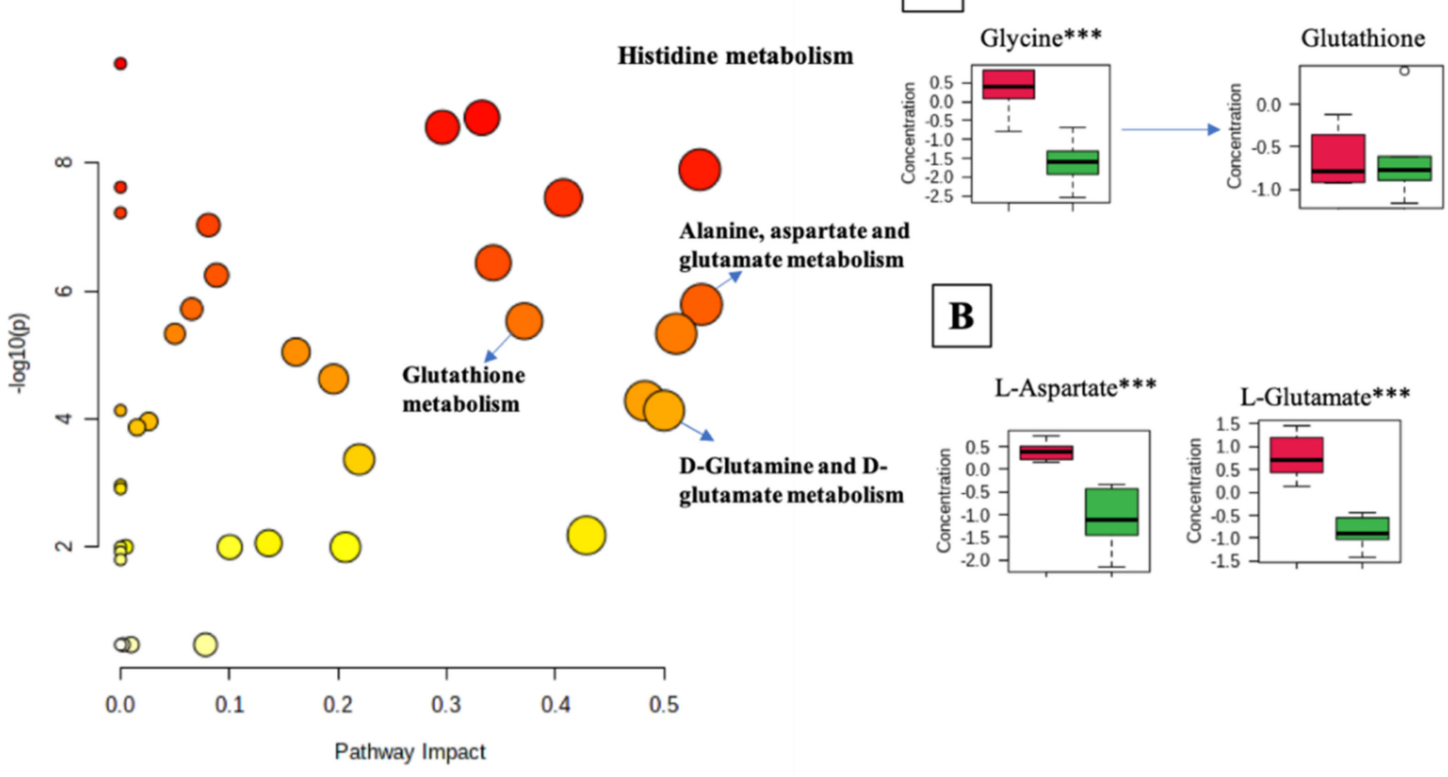

Figure 6. Pathway analysis associated with CTRL or CTRL_LPS of the RAW 264.7 cell $\operatorname{line}(\log (p)$ values from pathway enrichment analysis vs. values of the pathway impact). (A) Summary of relevant nodes in the pathway associated with the glutathione metabolism. (B) Summary of relevant nodes in the pathway associated with the D-glutamine and D-glutamate metabolism. Red boxplots-CTRL, green boxplots-CTRL_LPS. ${ }^{* * *} p<0.001$.

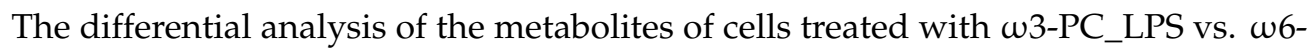
PC_LPS was associated with 22 metabolic pathways (FDR $<0.05$ ) including the alanine, aspartate, and glutamate metabolism ( $p$-value $=0.00015$, FDR $<0.01)$; D-glutamine and D-glutamate metabolism $(p$-value $=0.0005$, FDR $<0.01)$; and histidine metabolism $(p$-value $=0.0095$, FDR $<0.05)$, (Figure 7 and Supplementary Table S7). The boxplots of Figure 7A-C show statistically significant differences observed in the alanine and aspartate metabolism, where L-alanine and L-aspartate were significantly increased under w6-PC_LPS conditions compared to $\omega 3$-PC_LPS conditions. In addition, in the metabolism of D-glutamine and D-glutamate, L-glutamate and succinate were significantly increased under $\omega 6-$ PC _LPS conditions compared to $\omega 3$-PC_LPS conditions. 


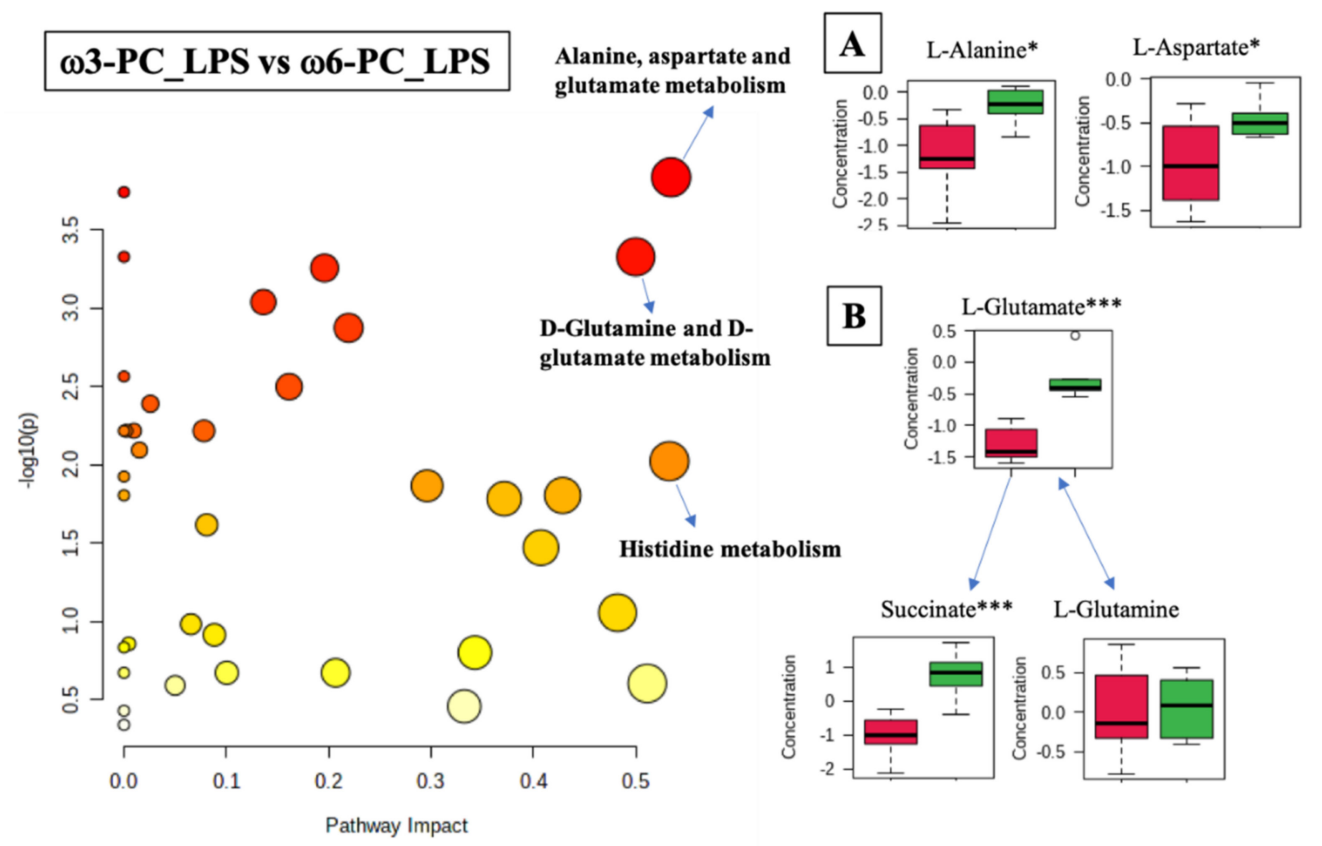

Figure 7. Pathway analysis associated with $\omega 3$-PC or $\omega 6-P C$ with LPS supplementation and stimulation of the RAW 264.7 cell $\operatorname{line}(\log (p)$ values from pathway enrichment analysis vs. values of the pathway impact). (A) Summary of relevant nodes in the pathway associated with the metabolism of alanine, aspartate, and glutamate. (B) Summary of relevant nodes in the pathway associated with the metabolism of D-glutamine and D-glutamate. Red boxplots w3-PC_LPS, green boxplots $\omega 6$-PC_LPS. ${ }^{*} p<0.05$ and ${ }^{* * *} p<0.001$.

\section{Discussion}

Intense research has been carried out on the biological activity of PUFAs, in particular on their contribution to the modulation of inflammatory responses and their impact on immune cells. The classic dichotomous classification of w3-PC as anti-inflammatory and $\omega 6-\mathrm{PC}$ as pro-inflammatory has been challenged by numerous studies, indicating that the impact of these bioactive lipids on immune cells is much more complex. In the present work, we sought, through a multi-omic approach, to explore the effects of two phospholipids containing $\omega 3$ and $\omega 6$ PUFAs (PC 18:0/22:6 or PC 18:0/20:4) on the macrophage's proteome, lipidome, and metabolome under resting and LPS-activated conditions.

In this study, the Griess reaction was used to estimate the total NO concentration in the culture medium and to assess the activation/inflammatory state of macrophages triggered by LPS and the modulation caused by $\omega 3$ and $\omega 6$ PLs supplementation. Although several more sensitive techniques can be used to measure nitrite, including fluorometric, electrochemical, and 3-nitrotyrosine quantification assays [32], the classical Griess spectrophotometric assay is most frequently used. In this experimental in vitro model of inflammation, $\mathrm{NO}$ levels reached $0.6-34 \mu \mathrm{M}$ in the culture medium and these values are within the detection range of the Griess reaction [33]. Our results demonstrate that $\omega 3-\mathrm{PC}$ and $\omega 6-\mathrm{PC}$ inhibit the lipopolysaccharide-stimulated production of nitric oxide. This decrease in NO production caused by $\omega 3-\mathrm{PC}$ and $\omega 6-\mathrm{PC}$ is not expected to be the main mechanistic cause of the observed effects of PCs on the macrophage lipidome, metabolome, and proteome. Other studies, for example using NOS inhibitors, will clarify the role of NO in this process.

Our results demonstrated that the supplementation of macrophages with $\omega 3$-PC or $\omega 6-$ PC has a distinct impact on the reprogramming of the proteome, lipidome, and metabolome. Regarding the number of molecular species found to be modulated, w6-PC supplementation caused the most pronounced effects on the three omic profiles analysed. However, if we focus on the analysis of the 50 most significantly modulated proteins, $\omega 3$-PC is clearly responsible for a pronounced impact, causing increased expression in almost all of the 
datasets. Among the proteins strongly upregulated by $\omega 3-\mathrm{PC}$, were tyrosine-protein kinase CSK (P41241), neutrophil cytosol factor 1 (Q09014), catalase (P24270), and several enzymes involved in lipid metabolism. Tyrosine-protein kinase CSK is a protein that downregulates the pro-inflammatory cytokine interleukin- 6 and TNF- $\alpha$, as well as the TLR-4-mediated activation of the ERK and p38 signalling pathways [34]. Neutrophil cytosol factor 1 (Ncf1), also known as p47(phox), is a subunit of the NADPH oxidase complex, being involved in the ROS biosynthetic process and in the regulation of the respiratory burst during inflammatory responses $[35,36]$. In turn, catalase is a detoxifying enzyme that neutralises ROS, mainly hydrogen peroxide $\left(\mathrm{H}_{2} \mathrm{O}_{2}\right)$, thus assuming an important role in cellular protection against oxidative damage [37]. Catalase has other anti-inflammatory roles, as it is also involved in the downregulation of NF- $\mathrm{kB}$ activity during a respiratory burst in macrophages, as well as in the upregulation of the PI3K signalling pathway [38,39]. The PI3K pathway has been reported to negatively modulate the MAPK signalling cascades, namely ERK1/2, p38, JNK, and the NF- $\mathrm{kB}$ pathway, and its activation has been demonstrated to be required for macrophage polarisation through an M2 phenotype [40-42].

Among the proteins related to lipid metabolism induced by $\omega 3-\mathrm{PC}$, acetyl-CoA acetyltransferase (Q8QZT1), 3-ketoacyl-CoA thiolase (Q8BWT1), and the trifunctional enzyme subunit alpha (Q8BMS1) may contribute to the $\omega 3$ PUFAs anti-inflammatory properties. These three proteins are involved in fatty acid $\beta$-oxidation (FAO), a process by which fatty acids are broken down into acetyl-CoA, which is also used in other metabolic pathways, such as the tricarboxylic acid (TCA) cycle or the oxidative phosphorylation (OXPHOS), to produce energy [43]. Recent studies have suggested that the polarisation of macrophages into M2-type (anti-inflammatory) is linked to an increase in the FAO pathway [44]. However, the role of FAO in the polarisation of macrophages is not entirely clear, as FAO has also been implicated in the activation of the NLRP3 inflammasome in M1-like macrophages, increasing the secretion of the pro-inflammatory cytokine IL-1 $\beta$ [45].

Regarding the impact of $\omega 6$-PC on the proteome of macrophages, the most significant changes found included the downregulation of proteins, such as aspartate aminotransferase (P05202), fumarate hydratase (P97807), and isocitrate dehydrogenase (P70404). Aspartate aminotransferase is a protein involved in the malate-aspartate shuttle that is also responsible for glutamate metabolism and converts L-aspartate into L-glutamate [46]. Isocitrate dehydrogenase is a key enzyme involved in the conversion of isocitrate to $\alpha$-ketoglutarate in the TCA cycle. Its decreased activity or expression is expected to result in decreased formation of $\alpha$-ketoglutarate, a key metabolite for the alternative (M2) activation of macrophages [47]. Finally, fumarate hydratase catalyses the reversible hydration/dehydration of fumarate to malate, which are intermediates of the TCA cycle [48]. It has been reported that the inhibition or decreased activity of fumarate hydratase leads to accumulation of fumarate and increased succinate levels [49,50]. High levels of fumarate inhibit the hypoxia-inducible factors (HIF) prolyl hydroxylases, leading to HIF-1 $\alpha$ stabilisation, which results in increased transcription of inflammatory and immune-related genes [51]. It has been demonstrated that the stabilisation of HIF- $1 \alpha$ is directly involved in the upregulation of the macrophage M1 markers [52,53]. High levels of fumarate also result in the succination of cysteinecontaining proteins, such as the kelch-like EC-associated protein 1 (KEAP1), inactivating it and impairing the expression of NRF2-ARE-dependent genes, such as HMOX1 and NQO1, which are involved in the cytoprotective and antioxidant response [54]. In turn, the accumulation of succinate has been demonstrated to play an important role in inflammation by enhancing IL-1 $\beta$ production through a HIF-1 $\alpha$ stabilisation-dependent mechanism [55]. However, recent reports demonstrate that the immunomodulatory functions of succinate are more complex, as it can suppress the secretion of the inflammatory mediators IL-6, tumours TNF- $\alpha$ and NO, as well as inhibit IL-1b mRNA transcription in inflammatory macrophages [56]. In addition, the silencing of SUCNR1, the receptor for succinate, results in the upregulation of the pro-inflammatory genes IL-1 $\beta$, IL-6, and IL-12b [57].

In contrast to the effects on the proteome, our results indicate that supplementation with $\omega 3-\mathrm{PC}$ and $\omega 6-\mathrm{PC}$ had a modest impact on the lipidome and metabolome of resting 
macrophages. However, in M1-like LPS-activated macrophages, profound alterations were observed in the three omic profiles of $\omega 3-\mathrm{PC}$ and $\omega 6-\mathrm{PC}$ co-treated cells. At the protein level, the most striking effects were observed in a group of proteins whose LPS-induced expression is further exacerbated by culture with PUFAs, in particular by w3-PC. Among the highly upregulated proteins, we found heme oxygenase-1 (P14901) NADPH-cytochrome P450 reductase (P37040), immunity-related GTPase family M protein 1 (Irgm1) (Q60766), sequestosome-1 (Q64337), and pleckstrin (Q9JHK5). This potentiation by PC-PUFAs of the expression of these proteins in M1 macrophages could contribute to w3-PC and w6-PC anti-inflammatory potential via multiple mechanisms. Heme oxygenase- 1 catalyses the NADPH-cytochrome P450 reductase-dependent metabolisation of the pro-oxidant heme to the antioxidant biliverdin and CO. These proteins, therefore, have important antioxidant and anti-inflammatory actions by inhibiting the secretion of the PAMP-induced pro-inflammatory cytokine while increasing the production of IL-10 in macrophages [58].

Irgm1 is involved in the innate immune response and is canonically induced by interferons-beta and gamma [59]. Previous studies have demonstrated that Irgm1 negatively regulate the production of pro-inflammatory cytokines mediated by TLR-4 in LPS-stimulated macrophages [60]. Mice lacking this protein demonstrate uncontrolled production of cytokines, contributing to excessive inflammation. Sequestosome-1 (SQSTM1), also known as the ubiquitin-binding protein p62, is central in autophagy processes and is also involved in the downregulation of NF- $\mathrm{kB}$ signalling, impairing the expression of TNF- $\alpha$, IL-1 $\beta$, IL-6, and IFN- $\beta$ [61]. Regarding pleckstrin, it is an abundant protein in platelets and leukocytes, where it affects the second messenger-based signalling events mediated by phospholipase C, PI3K $\gamma$, and inositol 5-phosphatases $[62,63]$.

This PUFAs-induced expression of pleckstrin and its effects on the metabolic process of phosphatidylinositol resulted in the increased levels of PIs observed in our lipidomic analysis. PIs are precursors of signalling molecules, such as phosphatidylinositol phosphates (PIPs), which play a central role in the activation of the PI3K-Akt pathway. As the PI3K-Akt pathway critically restricts proinflammatory responses in TLR-stimulated macrophages, it is plausible to hypothesise that the increased formation of PI mediated by $\omega 3$-PC and $\omega 6-\mathrm{PC}$ is one mechanism by which these active lipids exert anti-inflammatory effects.

The role of phospholipids in inflammation is increasingly recognised, with both anti-inflammatory and pro-inflammatory effects in macrophages and other immune cells being reported. Consistent with previous work, we found that the LPS-treatment of macrophages resulted in a profound lipidome remodelling with an emphasis on the increase of lipids classes canonically associated with inflammation, such as PC, LPC, LPE, Cer, and CL [64-66]. In addition to the aforementioned increase in PI production triggered by $\omega 3$-PC and $\omega 6$-PC supplementation, our data demonstrated that these PUFAs decreased the relative abundance of LPE and CL in LPS-stimulated macrophages, thereby contributing to a less pronounced inflammatory state. LPE arises from the hydrolysis of PE by phospholipase A2 (PLA2), its inflammatory actions being potentially due to the increased intracellular calcium levels via activation of PKC $[67,68]$. Regarding cardiolipins, under homeostatic conditions they are found exclusively in the inner membrane of mitochondria; however, under PAMP stimulation they translocate to the outer mitochondrial membrane, thus activating the NLRP3 inflammasome, which leads to increased production of inflammatory cytokines IL-1 $\beta$ and IL-18 $[69,70]$. Despite the referred changes induced by $\omega 3$-PC and $\omega 6$-PC in the lipidome of LPS-activated macrophages that may contribute to some extent to limiting their inflammatory state, we also observed alterations that may aggravate inflammation. It should be noted that the treatment with w3-PC and w6-PC of the LPS-activated macrophages resulted in increased levels of LPCs. LPCs are produced by PLA2-mediated hydrolysis of PC species and in macrophages have been demonstrated to promote M1 polarisation through TLR-mediated signalling and to increase phagocytic activity via AMPK and p38 MAPK-dependent mechanisms [71-73].

It has been demonstrated that the treatment of macrophages with LPS promotes a pronounced metabolic switch from oxidative phosphorylation toward glycolysis [74]. 
Paradoxically, despite the decrease in mitochondrial respiration, LPS treatment causes a strong increase in the TCA cycle intermediates fumarate, malate, and succinate. Succinate is transported from the mitochondria, through the dicarboxylic acid transporter to the cytosol, where it stabilises HIF-1 $\alpha$, boosting LPS-induced IL-1 mRNA transcription [53]. Moreover, succinate oxidation via mitochondrial succinate dehydrogenase has also been reported as a key event in the repropose of macrophage mitochondria from ATP synthesis to ROS production [75]. In these works, glutamine-dependent anaplerosis was found to be the major source of succinate. This is corroborated by our results, where we observed a significant decrease of glutamate in LPS-stimulated macrophages. Glutamine is converted to glutamate, which is then consumed to form $\alpha$-ketoglutarate and then succinate.

Regarding the impact of $\omega 3-\mathrm{PC}$ and $\omega 6-\mathrm{PC}$ on the metabolome of resting and LPSactivated macrophages, it demonstrated a behaviour similar to that observed for the lipidome profile: PUFAs minimally affected resting cells but strongly impacted the metabolome of LPS-activated macrophages. Compared to the $\omega 3$-PC respective conditions, the $\omega 6$-PC supplemented LPS-activated cells demonstrated significant increases in L-alanine, L-aspartate, L-glutamate, and succinate. As there was no difference in glutamine levels between cells exposed to $\omega 3-\mathrm{PC}$ and $\omega 6-\mathrm{PC}$, we hypothesise that the increase in glutamate triggered by $\omega 6$-PC may result from the transamination of $\alpha$-ketoglutarate. We also observed that $\omega 6-\mathrm{PC}$ decreases the expression of isocitrate dehydrogenase, the enzyme involved in the conversion of isocitrate to $\alpha$-ketoglutarate in the TCA cycle. Therefore, both of these events may lead to a substantial decrease in $\alpha$-ketoglutarate levels, skewing macrophages towards a more pronounced M1 phenotype $[49,50]$. Concerning succinate, its accumulation in macrophages supplemented with $\omega 6$-PC treated with LPS could result from the observed downregulation of fumarate hydratase protein expression triggered by w6-PC. It is unclear whether the accumulation of succinate induced by $\omega 6-\mathrm{PC}$ contributes to exacerbating the inflammatory status in M1-activated macrophages or, on the contrary, if it downmodulates their activation due to the pro- and anti-inflammatory properties that have been attributed to this metabolite [55-57].

Overall, the data from our multi-omic approach were congruent, demonstrating the complex metabolic network underlying the effects of $\omega 3$ and $\omega 6$ on the proteome, lipidome, and metabolome of M1-polarised macrophages. Although the results obtained support the established notion that $\omega 3$ PUFAS have superior anti-inflammatory activity compared with $\omega 6$ PUFAs, they also evidence that neither $\omega 3$ nor $\omega 6$ have exclusively anti- or pro-inflammatory activities, respectively.

\section{Materials and Methods}

\subsection{Chemicals and Reagents}

Acetonitrile (ACN) was purchased from Merck (Darmstadt, Germany), the phospholipids were purchased from Avanti Lipids Polar (Alabaster, AL, USA), and the remaining chemicals / reagents were purchased from Sigma-Aldrich (St Louis, MO, USA). All chemicals were of analytical grade and milli-Q water was used throughout all experiments.

\subsection{Cell Culture}

The mouse leukemic monocyte-macrophage cell line RAW 264.7 (ATCC TIB-71, American Tissue Culture Collection, Manassas, VA, USA), was maintained in high-glucose Dulbecco's Modified Eagle Medium (DMEM), supplemented with 10\% non-inactivated foetal bovine serum (FBS), streptomycin $(100 \mu \mathrm{g} / \mathrm{mL})$ and penicillin $(100 \mathrm{U} / \mathrm{mL}), 1.5 \mathrm{~g} / \mathrm{L}$ sodium bicarbonate, and $2 \mathrm{mM}$ of glutamine. The cells were kept in the culture in an incubator at $37{ }^{\circ} \mathrm{C}$ and $5 \% \mathrm{CO}_{2}$ and were sub-cultured every $2-3$ days to maintain cell density between 0.5 and $0.8 \times 10^{6}$ cells $/ \mathrm{mL}$.

\subsection{Cell Viability Assay}

To determine the impact of the phospholipids on cell viability, the resazurin reduction assay was performed accordingly [76]. Macrophages were seeded at 40,000 cells/well in 
a 96-well plate and allowed to stabilise in the incubator for a period of $24 \mathrm{~h}$. Afterwards,

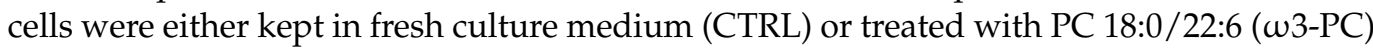

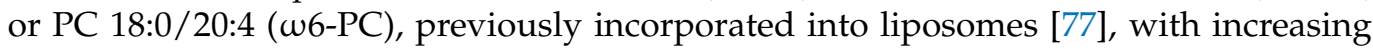
concentrations of phospholipid $(10,20,50,100$, and $200 \mu \mathrm{M})$. After $21 \mathrm{~h}$ of incubation, cells $(n=3)$ were treated with resazurin $(50 \mu \mathrm{M})$ and incubated for $3 \mathrm{~h}$ and after $24 \mathrm{~h}$, resorufin was quantified on a plate reader (Multiskan GO 1510-00111C, ThermoScientific, Waltham, MA, USA). All assays were performed in triplicate.

\subsection{Quantitative Analysis of Nitrites by Griess Reaction}

To assess the anti-inflammatory activity of the phospholipids, nitric oxide production was determined by quantitative analysis of nitrites with Griess reagent, as previously described [78]. Macrophages were seeded at 40,000 cells/well in a 96-well plate and allowed to stabilise for a period of $24 \mathrm{~h}$. Cells were either kept in fresh culture medium

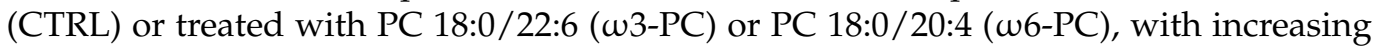
concentrations of phospholipids $(10,20,50,100$, and $200 \mu \mathrm{M})$. After $1 \mathrm{~h}$ of incubation, LPS was added as a positive control for inflammatory activity at a final concentration of $100 \mathrm{ng} / \mathrm{mL}$. Phospholipid incubation was carried out for a period of $24 \mathrm{~h}$. All assays were performed in triplicate.

\subsection{Incubation with Phospholipids}

For phospholipid incubation assays, macrophages were seeded in 6-well culture plates at $1 \times 10^{6}$ cells $/ \mathrm{mL}$ per well. After $24 \mathrm{~h}$, cells were either maintained in a fresh culture

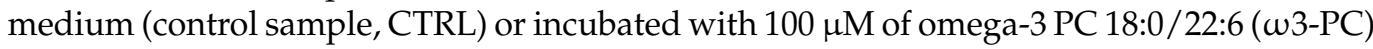

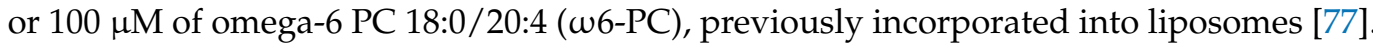
After $1 \mathrm{~h}$ of phospholipid incubation, one sample of each condition (CTRL, w3-PC, and w6-PC) was further treated with $100 \mathrm{ng} / \mathrm{mL}$ of lipopolysaccharide (LPS) as a positive control for inflammatory activity (resulting in CTRL_LPS, w3-PC_LPS, and w6-PC_LPS samples, respectively). Incubation with phospholipids was performed for $24 \mathrm{~h}$ periods. In the case of proteomics analysis, all experiments with phospholipid incubation were carried out using free serum medium.

\subsection{Proteomics}

The proteins were extracted using RIPA buffer. The pellets were resuspended in RIPA solution supplemented with 1M DTT and anti-proteases cocktail (P8340, SigmaAldrich), vortexed, and kept on ice for $15 \mathrm{~min}$. Samples were centrifuged at $14,000 \times g$ for $15 \mathrm{~min}\left(4^{\circ} \mathrm{C}\right)$ and the resulting supernatants were carefully collected. The cell lysates were stored at $-80^{\circ} \mathrm{C}$. Protein concentrations were determined using the $\mathrm{RC} / \mathrm{DC}$ protein assay kit (BioRad, Hercules, CA, USA) according to the manufacturer's protocol. Denaturing SDS-PAGE and in-gel digestion was performed as previously described [79].

The tryptic peptide samples that resulted from in-gel digestion were reconstituted in $40 \mu \mathrm{L}$ of $5 \%$ ACN with $0.1 \%$ FA in liquid chromatography-mass spectrometry (LC-MS)grade water and loaded onto an EASY-Spray high performance-liquid chromatography (HPLC) column $(75 \mu \mathrm{m} \times 150 \mathrm{~mm}, 2 \mu \mathrm{m}, 100$ A; Thermo Fisher Scientific, Bremen, Germany) operated at $40^{\circ} \mathrm{C}$. Nanoflow LC-MS/MS was performed on a Q-Exactive hybrid quadrupole Orbitrap mass spectrometer (Thermo Fisher Scientific, Bremen, Germany) coupled to an HPLC system (Ultimate 3000 Dionex; Thermo Fisher Scientific, Bremen, Germany). The buffer system consisted of two mobile phases, buffer A $(0.1 \%$ FA in HPLC grade $\left.\mathrm{H}_{2} \mathrm{O}\right)$ and buffer $\mathrm{B}\left(80 \%\right.$ HPLC grade $\mathrm{ACN}$ in $20 \% \mathrm{HPLC}$ grade $\mathrm{H}_{2} \mathrm{O}$ and $\left.0.1 \% \mathrm{FA}\right)$ at a flow rate of $300 \mathrm{~nL} / \mathrm{min}$. Peptides were eluted with a linear gradient of $5-24 \%$ B over $50 \mathrm{~min}$, increased to $36 \% \mathrm{~B}$ over $10 \mathrm{~min}$ and held for $5 \mathrm{~min}$. The mass spectrometer was operated in the positive ion mode (electrospray voltage $2.0 \mathrm{kV}$ ) with a capillary temperature of $250{ }^{\circ} \mathrm{C}$. MS survey scans were performed with a resolution of 70,000, automatic gain control (AGC) target $1 \times 10^{6}$, and maximum injection time (IT) $100 \mathrm{~ms}$. The 10 most intense peaks per MS scan were selected for higher-energy collisional dissociation (HCD) MS/MS 
experiments with a resolution of 17,500 , AGC target of $5 \times 10^{4}$, maximum IT of $50 \mathrm{~ms}$, a normalised collision energy $\mathrm{y}^{\mathrm{TM}}(\mathrm{CE})$ of 28 , isolation width $1.2 \mathrm{Th}$, and dynamic exclusion of $30 \mathrm{~s}$. Data acquisition was performed using the Xcalibur data system v3.3 (Thermo Fisher Scientific, Bremen, Germany).

Proteins were identified using the Proteome Discoverer software (v2.2.0.388, Thermo Fisher Scientific, Bremen, Germany). The MS data were searched against the Mus musculus (mouse) protein database (Accessed on 9 June 2021, Taxon identifier = 10,090, Swiss Prot), with SEQUEST HT and MS AMANDA 2.0 search engines, and with percolator validation. The search parameters were as follows: carbamidomethylation of cysteine $(C)$ was defined as a static modification and oxidation of methionine (M) and acetylation of N-terminal protein (N-Terminus) were set as dynamic modifications. The mass tolerance of precursor and fragment ions was 10 ppm (MS1) and $0.02 \mathrm{Da}$ (MS2). Up to two missed cleavages were considered for trypsin digestion. The threshold of the global false discovery rate (FDR) for peptides and proteins was set at 0.01 . Only proteins with a minimum number of two unique peptides and with a peptide length no lower than 6 amino acids were considered. Proteins with low variability were removed from the analysis as artefacts. Gene ontology (GO) analysis was performed on QuickGO (https: / / www.ebi.ac.uk/QuickGO/ (accessed on 9 June 2021)) (EMBL-EBI, Cambridgeshire, UK), assigning the protein's molecular functions, according to the Gene Ontology database.

\subsection{Lipidomics}

Total lipids were extracted from cells and harvested as described for the proteomics analysis using the Bligh and Dyer extraction method, and total phospholipids concentration was determined using the phosphorous assay, as described previously [80,81].

Samples (10 $\mu \mathrm{L}$ of each sample (equivalent to $10 \mu \mathrm{g}$ of phospholipid), $82 \mu \mathrm{L}$ of eluent (95\% of mobile phase B and $5 \%$ of mobile phase A), and $8 \mu \mathrm{L}$ ) were mixed with $8 \mu \mathrm{L}$ of a solution of PL standards (dimyristoyl-phosphatidylcholine (dMPC)-0.02 $\mu \mathrm{g}$, dimyristoylphosphatidylethanolamine (dMPE)-0.02 $\mu \mathrm{g}$, Sphingomyelin (SM) (d18:1/17:0)-0.02 $\mu \mathrm{g}$, Lysophosphatidylcholine (LPC) (19:0)-0.02 $\mu \mathrm{g}$, dipalmitoyl-phosphoinositol (dPPI)-0.02 $\mu$ g, cardiolipin (CL) (14:0)4-0.02 $\mu \mathrm{g}$, dimyristoyl-phosphatidylglycerol (dMPG)-0.012 $\mu \mathrm{g}$, dimyristoyl-Phosphatidic acid (dMPA)-0.02 $\mu \mathrm{g}$, ceramide (Cer) (d18:1/17:0)-0.02 $\mu \mathrm{g}$, dimyristoyl-phosphatidylserine (dMPS)-0.02 $\mu \mathrm{g}$ (Avanti Polar Lipids, Inc., Alabaster, AL, USA)). This mixture $(5 \mu \mathrm{L})$ was injected into the Ascentis ${ }^{\circledR}$ Express microbore column $(10 \mathrm{~cm} \times 2.1 \mathrm{~mm}, 2.7 \mu \mathrm{m}$; SigmaAldrich, Saint Louis, MO, USA) at $35^{\circ} \mathrm{C}$ and a flow-rate of $200 \mu \mathrm{L}$ min -1 and analysed by hydrophilic interaction liquid chromatography-mass spectrometry (HILIC-LC-MS) on an Ultimate 3000 Dionex ultra-high-performance liquid chromatography (UHPLC) system (Thermo Fisher Scientific, Bremen, Germany) coupled online to an orbitrap Q-Exactive mass spectrometer (Thermo Fisher Scientific, Bremen, Germany), as previously described [82].

Lipid species were identified using mass spectrometry-data independent analysis (MSDIAL) v4.70 software [83]. Identification of the lipid species was performed in the negative and positive ionisation modes, using the raw files acquired in MS/MS mode, and converted by the ABF converter (https: / / www.reifycs.com/AbfConverter / (accessed on 5 July 2021)) against the lipid database provided by the MS-DIAL software. The tolerances for MS and MS/MS search were set at $0.01 \mathrm{Da}$ and $0.05 \mathrm{Da}$, and all identifications were manually verified. The validated species were integrated and quantified in the MZmine v2.53 software [84]. Raw LC-MS files were subjected to smoothing and filtering methods, peak detection (including chromatogram construction, peak deconvolution and deisotoping) and peak alignment with gap filling. Later, the integrated peak areas of the extracted ion chromatograms (XIC) were exported, and normalisation was obtained by calculating the ratio against a selected internal lipid standard with the closest retention time.

\subsection{Metabolomics}

The dried samples taken from the aqueous phase of the Bligh and Dyer extraction method were resuspended in an ice-cold solution of MeOH: ACN: Mili-Q water (4:4:2) solu- 
tion [85]. The samples were then kept at $-80^{\circ} \mathrm{C}$ for $20 \mathrm{~min}$ and centrifuged at $14,000 \times g$ for 10 min at $4-8{ }^{\circ} \mathrm{C}$ (B. Braun Biotech International GmbH, Berlin, Germany). The supernatants were collected and dried under a vacuum. Later, dried samples were resuspended in $80 \%$ MS grade MeOH containing an internal standard (Leu-Tyr, $0.02 \mathrm{mg} / \mathrm{mL}$, Sigma-Aldrich, Saint Louis, MO, USA). Samples were normalised by the volume corresponding to the PL concentration measured from the phosphorous assay $(0.1 \mu \mathrm{g} / \mu \mathrm{L})$.

Metabolites were analysed by HILIC-LC-MS, under the same instrumental setup as described previously for lipidomics analysis, except for the HPLC programme and MS/MS acquisition conditions. The solvent system consisted of two mobile phases, a mobile phase A (ACN: MeOH: H2O, 2.5:2.5:95 (per volume) with $5 \mathrm{mM}$ ammonium acetate and $0.1 \%$ of $\mathrm{FA}$ ) and mobile phase B (ACN:MeOH, 90:10 (per volume) with $5 \mathrm{mM}$ ammonium formate and $0.1 \%$ of FA). Metabolites were eluted with a $100 \%$ of mobile phase B for $1 \mathrm{~min}$, followed by a linear gradient of $0-50 \%$ A over $15 \mathrm{~min}$ and held for $5 \mathrm{~min}$. The mass spectrometer operated in the positive $(3.1 \mathrm{kV})$ and negative $(-2.8 \mathrm{kV})$ ion modes with a capillary temperature of $350{ }^{\circ} \mathrm{C}$ and a sheath gas flow of $20 \mathrm{U}$. MS survey scans were performed with a resolution of 70,000, AGC target $1 \times 10^{6}$, and maximum IT $100 \mathrm{~ms}$. Scan range between 65 to $900 \mathrm{~m} / \mathrm{z}$. The 10 most intense peaks per MS scan were selected for HCD MS/MS experiments with a resolution of 17,500, AGC target of $1 \times 10^{3}$, and maximum IT of 50 ms; a normalised collision energy ${ }^{\mathrm{TM}}$ of 20,30, and 40; isolation width $1.5 \mathrm{Th}$; and dynamic exclusion of $30 \mathrm{~s}$. Quality control (QC) samples were included and prepared by pooling aliquots of all samples. Data acquisition was performed using the Xcalibur data system (v3.3, Thermo Fisher Scientific, Waltham, MA, USA).

For the processing of LC-MS data, the workflow was similar to the lipidomics data analysis. Metabolites were identified using MS-DIAL v4.70 software [83], against databases. Identification of metabolites was performed in negative and positive ionisation modes, using the QC raw files acquired in MS/MS analysis for each mode. The validated species were integrated and quantified, using MZmine v2.53 software [84]. Normalisation of the integrated peak areas of the XIC was achieved by calculating the ratio against the internal standard.

\subsection{Statistical Analysis}

Multivariate and univariate analyses were performed using R v3.6.2 [86] in Rstudio v1.2.5 [87]. The data from proteomics and lipidomics analysis were log-transformed, and the EigenMS was normalised [88] and subjected to auto-scaling. Metabolomics data were normalised by sum before being log-scaled, and the EigenMS was normalised [88] and subjected to auto-scaling. The missing values were replaced by $1 / 5$ of the minimum positive values of their corresponding variables. The principal component analysis (PCA) was performed for all the omics, with the $\mathrm{R}$ built-in function and the $\mathrm{R}$ package pcaMethods [89]. In univariate analysis, the Kruskal-Wallis test followed by Dunn 's post hoc test was used for nonparametric comparisons and pairwise multiple-comparison between the 6 conditions, respectively. PCA graphs and boxplots were created using the R package ggplot2 [90], and heatmaps were created using the R package pheatmap, using "Euclidean" as the clustering distance, and "ward.D" as the clustering method [91]. To explore the metabolomic profiles, we also applied the pathway analysis module from Metaboanalyst (https:/ / www.metaboanalyst.ca (accessed on 12 July 2021)) [92], using the following pathway analysis parameters: enrichment method-Global Test, topology analysis-relative-betweenness centrality; pathway library-Mus musculus (KEGG).

Supplementary Materials: The following supporting information can be downloaded at: https: //www.mdpi.com/article/10.3390/ijms23042139/s1. 


\begin{abstract}
Author Contributions: Data curation, S.A., S.G. and D.L.; formal analysis, T.M. (Tatiana Maurício), B.M.N. and R.D.; funding acquisition, R.D. and P.D.; investigation, T.M. (Tatiana Maurício), S.A. and S.G.; methodology, T.M. (Tânia Melo), R.D. and P.D.; project administration, P.D.; supervision, R.D and P.D.; validation, S.A., S.G., D.L. and T.M. (Tânia Melo); writing-original draft, T.M. (Tatiana Maurício) and B.M.N.; writing-review and editing, B.M.N., R.D. and P.D. All authors have read and agreed to the published version of the manuscript.
\end{abstract}

Funding: This research was funded by FCT/MCT with financial support to LAQV/REQUIMTE (UIDB/50006/2020), CESAM (UIDB/50017/2020+UIDP/50017/2020), and RNEM-Portuguese Mass Spectrometry Network (LISBOA-01-0145-FEDER-402-022125) through national funds and, where applicable, co-financed by the FEDER, within the PT2020 Partnership Agreement. This research was funded by the Polish National Agency for Academic Exchange (NAWA)/(PPI/APM/2018/00015/U/001) under the project "Preparation of omic tools for the diagnostics and assessment of efficacy of treatment of inflammatory diseases". This work has received funding under the project AlgaValor, from the Portugal 2020 program (grant agreement $n^{\circ}$ POCI-01-0247-FEDER-035234; LISBOA-01-0247-FEDER035234; ALG-01-0247-FEDER-035234). The authors are thankful to the COST Action EpiLipidNET, CA19105-Pan-European Network in Lipidomics and EpiLipidomics.

Data Availability Statement: The data that support the findings of this study are available from the corresponding author upon reasonable request.

Conflicts of Interest: The authors declare no conflict of interest.

\title{
References
}

1. Fujiwara, N.; Kobayashi, K. Macrophages in Inflammation. Curr. Drug Target-Inflamm. Allergy 2005, 4, 281-286. [CrossRef] [PubMed]

2. Zhang, X.; Mosser, D. Macrophage Activation by Endogenous Danger Signals. J. Pathol. 2008, 214, 161-178. [CrossRef] [PubMed]

3. Gutiérrez, S.; Svahn, S.L.; Johansson, M.E. Effects of Omega-3 Fatty Acids on Immune Cells. Int. J. Mol. Sci. 2019, 20, 5028. [CrossRef] [PubMed]

4. $\quad$ Laskin, D.L.; Sunil, V.R.; Gardner, C.R.; Laskin, J.D. Macrophages and Tissue Injury: Agents of Defense or Destruction? Annu. Rev. Pharmacol. Toxicol. 2011, 51, 267-288. [CrossRef] [PubMed]

5. Funes, S.C.; Rios, M.; Escobar-Vera, J.; Kalergis, A.M. Implications of Macrophage Polarization in Autoimmunity. Immunology 2018, 154, 186-195. [CrossRef] [PubMed]

6. Curren Smith, E.W. Macrophage Polarization and Its Role in Cancer. J. Clin. Cell. Immunol. 2015, 6, 338. [CrossRef]

7. Schett, G.; Neurath, M.F. Resolution of Chronic Inflammatory Disease: Universal and Tissue-Specific Concepts. Nat. Commun. 2018, 9, 3261. [CrossRef]

8. Radzikowska, U.; Rinaldi, A.O.; Çelebi Sözener, Z.; Karaguzel, D.; Wojcik, M.; Cypryk, K.; Akdis, M.; Akdis, C.A.; Sokolowska, M. The Influence of Dietary Fatty Acids on Immune Responses. Nutrients 2019, 11, 2990. [CrossRef]

9. Venter, C.; Meyer, R.W.; Nwaru, B.I.; Roduit, C.; Untersmayr, E.; Adel-Patient, K.; Agache, I.; Agostoni, C.; Akdis, C.A.; Bischoff, S.; et al. EAACI Position Paper: Influence of Dietary Fatty Acids on Asthma, Food Allergy, and Atopic Dermatitis. Allergy 2019, 74, 1429-1444. [CrossRef]

10. Yan, Z.; Gibson, S.A.; Buckley, J.A.; Qin, H.; Benveniste, E.N. Role of the JAK/STAT Signaling Pathway in Regulation of Innate Immunity in Neuroinflammatory Diseases. Clin. Immunol. 2018, 189, 4-13. [CrossRef]

11. Calder, P.C. Fatty Acids and Inflammation: The Cutting Edge between Food and Pharma. Eur. J. Pharmacol. 2011, 668, S50-S58. [CrossRef] [PubMed]

12. D'Angelo, S.; Motti, M.L.; Meccariello, R. $\omega-3$ and $\omega-6$ Polyunsaturated Fatty Acids, Obesity and Cancer. Nutrients $2020,12,2751$. [CrossRef] [PubMed]

13. Chang, H.Y.; Lee, H.-N.; Kim, W.; Surh, Y.-J. Docosahexaenoic Acid Induces M2 Macrophage Polarization through Peroxisome Proliferator-Activated Receptor $\gamma$ Activation. Life Sci. 2015, 120, 39-47. [CrossRef] [PubMed]

14. Kumar, N. 15-Lipoxygenase Metabolites of $\alpha$-Linolenic Acid, [13-(S)-HPOTrE and 13-(S)-HOTrE], Mediate Anti-Inflammatory Effects by Inactivating NLRP3 Inflammasome. Sci. Rep. 2015, 14, 1-14. [CrossRef]

15. Yan, Y.; Jiang, W.; Spinetti, T.; Tardivel, A.; Castillo, R.; Bourquin, C.; Guarda, G.; Tian, Z.; Tschopp, J.; Zhou, R. Omega-3 Fatty Acids Prevent Inflammation and Metabolic Disorder through Inhibition of NLRP3 Inflammasome Activation. Immunity 2013, 38, 1154-1163. [CrossRef]

16. Kawano, A.; Ariyoshi, W.; Yoshioka, Y.; Hikiji, H.; Nishihara, T.; Okinaga, T. Docosahexaenoic Acid Enhances M2 Macrophage Polarization via the P38 Signaling Pathway and Autophagy. J. Cell. Biochem. 2019, 120, 12604-12617. [CrossRef]

17. Mullen, A.; Loscher, C.E.; Roche, H.M. Anti-Inflammatory Effects of EPA and DHA Are Dependent upon Time and Dose-Response Elements Associated with LPS Stimulation in THP-1-Derived Macrophages. J. Nutr. Biochem. 2010, 7, 444-450. [CrossRef]

18. Zhao, Y.; Joshi-Barve, S.; Barve, S.; Chen, L.H. Eicosapentaenoic Acid Prevents LPS-Induced TNF- $\alpha$ Expression by Preventing NF-KB Activation. J. Am. Coll. Nutr. 2004, 23, 71-78. [CrossRef] 
19. Luo, W.; Xu, Q.; Wang, Q.; Wu, H.; Hua, J. Effect of Modulation of PPAR- $\gamma$ Activity on Kupffer Cells M1/M2 Polarization in the Development of Non-Alcoholic Fatty Liver Disease. Sci. Rep. 2017, 7, 44612. [CrossRef]

20. Oh, D.Y.; Talukdar, S.; Bae, E.J.; Imamura, T.; Morinaga, H.; Fan, W.; Li, P.; Lu, W.J.; Watkins, S.M.; Olefsky, J.M. GPR120 Is an Omega-3 Fatty Acid Receptor Mediating Potent Anti-Inflammatory and Insulin-Sensitizing Effects. Cell 2010, 142, 687-698. [CrossRef]

21. Kelley, D.S. Modulation of Human Immune and Inflammatory Responses by Dietary Fatty Acids. Nutrition 2001, 17, 669-673. [CrossRef]

22. Su, N.; Li, Y.; Wang, J.; Fan, J.; Li, X.; Peng, W.; Yu, X.; Zhou, T.-B.; Jiang, Z. Role of MAPK Signal Pathways in Differentiation Process of M2 Macrophages Induced by High-Ambient Glucose and TGF-B1. J. Recept. Signal Transduct. 2015, 35, 396-401. [CrossRef] [PubMed]

23. Chang, C.-S.; Sun, H.-L.; Lii, C.-K.; Chen, H.-W.; Chen, P.-Y.; Liu, K.-L. Gamma-Linolenic Acid Inhibits Inflammatory Responses by Regulating NF-KB and AP-1 Activation in Lipopolysaccharide-Induced RAW 264.7 Macrophages. Inflammation 2010, 33 , 46-57. [CrossRef] [PubMed]

24. Walloschke, B.; Fuhrmann, H.; Schumann, J. Enrichment of RAW264.7 Macrophages with Essential 18-Carbon Fatty Acids Affects Both Respiratory Burst and Production of Immune Modulating Cytokines. J. Nutr. Biochem. 2010, 21, 556-560. [CrossRef]

25. Schoeniger, A.; Adolph, S.; Fuhrmann, H.; Schumann, J. The Impact of Membrane Lipid Composition on Macrophage Activation in the Immune Defense against Rhodococcus Equi and Pseudomonas Aeruginosa. Int. J. Mol. Sci. 2011, 12, 7510-7528. [CrossRef]

26. De Lima, T.M.; de Sa Lima, L.; Scavone, C.; Curi, R. Fatty Acid Control of Nitric Oxide Production by Macrophages. FEBS Lett. 2006, 580, 3287-3295. [CrossRef]

27. Machado, R.M.; Nakandakare, E.R.; Quintão, E.C.R.; Cazita, P.M.; Koike, M.K.; Nunes, V.S.; Ferreira, F.D.; Afonso, M.S.; Bombo, R.P.A.; Machado-Lima, A.; et al. Omega-6 Polyunsaturated Fatty Acids Prevent Atherosclerosis Development in LDLr-KO Mice, in Spite of Displaying a pro-Inflammatory Profile Similar to Trans Fatty Acids. Atherosclerosis 2012, 224, 66-74. [CrossRef]

28. Gilroy, D.W.; Edin, M.L.; De Maeyer, R.P.H.; Bystrom, J.; Newson, J.; Lih, F.B.; Stables, M.; Zeldin, D.C.; Bishop-Bailey, D. CYP450-Derived Oxylipins Mediate Inflammatory Resolution. Proc. Natl. Acad. Sci. USA 2016, 113, E3240. [CrossRef]

29. Brouwers, H.; Jónasdóttir, H.S.; Kuipers, M.E.; Kwekkeboom, J.C.; Auger, J.L.; Gonzalez-Torres, M.; López-Vicario, C.; Clària, J.; Freysdottir, J.; Hardardottir, I.; et al. Anti-Inflammatory and Proresolving Effects of the Omega-6 Polyunsaturated Fatty Acid Adrenic Acid. J. Immunol. 2020, 205, 2840-2849. [CrossRef]

30. Farvid, M.S.; Ding, M.; Pan, A.; Sun, Q.; Chiuve, S.E.; Steffen, L.M.; Willett, W.C.; Hu, F.B. Dietary Linoleic Acid and Risk of Coronary Heart Disease: A Systematic Review and Meta-Analysis of Prospective Cohort Studies. Circulation 2014, 130, 1568-1578. [CrossRef]

31. Wu, J.H.Y.; Marklund, M.; Imamura, F.; Tintle, N.; Ardisson Korat, A.V.; de Goede, J.; Zhou, X.; Yang, W.-S.; de Oliveira Otto, M.C.; Kröger, J.; et al. Omega-6 Fatty Acid Biomarkers and Incident Type 2 Diabetes: Pooled Analysis of Individual-Level Data for 39,740 Adults from 20 Prospective Cohort Studies. Lancet Diabetes Endocrinol. 2017, 5, 965-974. [CrossRef]

32. Bryan, N.S.; Grisham, M.B. Methods to Detect Nitric Oxide and Its Metabolites in Biological Samples. Free Radic. Biol. Med. 2007, 43, 645-657. [CrossRef] [PubMed]

33. Pinto, R.V.; Antunes, F.; Pires, J.; Silva-Herdade, A.; Pinto, M.L. A Comparison of Different Approaches to Quantify Nitric Oxide Release from NO-Releasing Materials in Relevant Biological Media. Molecules 2020, 25, 2580. [CrossRef] [PubMed]

34. Aki, D.; Mashima, R.; Saeki, K.; Minoda, Y.; Yamauchi, M.; Yoshimura, A. Modulation of TLR Signalling by the C-Terminal Src Kinase (Csk) in Macrophages. Genes Cells 2005, 10, 357-368. [CrossRef]

35. Pizzolla, A.; Hultqvist, M.; Nilson, B.; Grimm, M.J.; Eneljung, T.; Jonsson, I.-M.; Verdrengh, M.; Kelkka, T.; Gjertsson, I.; Segal, B.H.; et al. Reactive Oxygen Species Produced by the NADPH Oxidase 2 Complex in Monocytes Protect Mice from Bacterial Infections. J. Immunol. Baltim. Md 1950 2012, 188, 5003-5011. [CrossRef]

36. Mortimer, P.M.; Mc Intyre, S.A.; Thomas, D.C. Beyond the Extra Respiration of Phagocytosis: NADPH Oxidase 2 in Adaptive Immunity and Inflammation. Front. Immunol. 2021, 12, 3558. [CrossRef]

37. Glorieux, C.; Calderon, P.B. Catalase, a Remarkable Enzyme: Targeting the Oldest Antioxidant Enzyme to Find a New Cancer Treatment Approach. Biol. Chem. 2017, 398, 1095-1108. [CrossRef]

38. Jang, B.-C.; Kim, D.-H.; Park, J.-W.; Kwon, T.K.; Kim, S.-P.; Song, D.-K.; Park, J.-G.; Bae, J.-H.; Mun, K.-C.; Baek, W.-K.; et al. Induction of Cyclooxygenase-2 in Macrophages by Catalase: Role of NF-KB and PI3K Signaling Pathways. Biochem. Biophys. Res. Commun. 2004, 316, 398-406. [CrossRef]

39. Kaul, N.; Forman, H.J. Activation of NFkB by the Respiratory Burst of Macrophages. Free Radic. Biol. Med. 1996, 21, 401-405. [CrossRef]

40. Guha, M.; Mackman, N. The Phosphatidylinositol 3-Kinase-Akt Pathway Limits Lipopolysaccharide Activation of Signaling Pathways and Expression of Inflammatory Mediators in Human Monocytic Cells *. J. Biol. Chem. 2002, 277, 32124-32132. [CrossRef]

41. Günzl, P.; Bauer, K.; Hainzl, E.; Matt, U.; Dillinger, B.; Mahr, B.; Knapp, S.; Binder, B.R.; Schabbauer, G. Anti-Inflammatory Properties of the PI3K Pathway Are Mediated by IL-10/DUSP Regulation. J. Leukoc. Biol. 2010, 88, 1259-1269. [CrossRef] [PubMed]

42. Vergadi, E.; Ieronymaki, E.; Lyroni, K.; Vaporidi, K.; Tsatsanis, C. Akt Signaling Pathway in Macrophage Activation and M1/M2 Polarization. J. Immunol. 2017, 198, 1006. [CrossRef] [PubMed] 
43. Schulz, H. Beta Oxidation of Fatty Acids. Biochim. Biophys. Acta BBA-Lipids Lipid Metab. 1991, 1081, 109-120. [CrossRef]

44. Batista-Gonzalez, A.; Vidal, R.; Criollo, A.; Carreño, L.J. New Insights on the Role of Lipid Metabolism in the Metabolic Reprogramming of Macrophages. Front. Immunol. 2020, 10, 2993. [CrossRef]

45. Moon, J.-S.; Nakahira, K.; Chung, K.-P.; DeNicola, G.M.; Koo, M.J.; Pabón, M.A.; Rooney, K.T.; Yoon, J.-H.; Ryter, S.W.; StoutDelgado, H.; et al. NOX4-Dependent Fatty Acid Oxidation Promotes NLRP3 Inflammasome Activation in Macrophages. Nat. Med. 2016, 22, 1002-1012. [CrossRef]

46. Ellinger, J.J.; Lewis, I.A.; Markley, J.L. Role of Aminotransferases in Glutamate Metabolism of Human Erythrocytes. J. Biomol. NMR 2011, 49, 221-229. [CrossRef]

47. Liu, P.-S.; Wang, H.; Li, X.; Chao, T.; Teav, T.; Christen, S.; Di Conza, G.; Cheng, W.-C.; Chou, C.-H.; Vavakova, M.; et al. $\alpha$-Ketoglutarate Orchestrates Macrophage Activation through Metabolic and Epigenetic Reprogramming. Nat. Immunol. 2017, 18, 985-994. [CrossRef]

48. Woods, S.A.; Schwartzbach, S.D.; Guest, J.R. Two biochemically distinct classes of fumarase in Escherichia coli. Biochimica Biophysica Acta 1988, 954, 14-26. [CrossRef]

49. Tyrakis, P.A.; Yurkovich, M.E.; Sciacovelli, M.; Papachristou, E.K.; Bridges, H.R.; Gaude, E.; Schreiner, A.; D'Santos, C.; Hirst, J.; Hernandez-Fernaud, J.; et al. Fumarate Hydratase Loss Causes Combined Respiratory Chain Defects. Cell Rep. 2017, 21, 1036-1047. [CrossRef]

50. Pollard, P.; Wortham, N.; Tomlinson, I. The TCA Cycle and Tumorigenesis: The Examples of Fumarate Hydratase and Succinate Dehydrogenase. Ann. Med. 2003, 35, 634-635. [CrossRef]

51. McGettrick, A.F.; O'Neill, L.A.J. The Role of HIF in Immunity and Inflammation. Cell Metab. 2020, 32, 524-536. [CrossRef] [PubMed]

52. Takeda, N.; O’Dea, E.L.; Doedens, A.; Kim, J.; Weidemann, A.; Stockmann, C.; Asagiri, M.; Simon, M.C.; Hoffmann, A.; Johnson, R.S. Differential Activation and Antagonistic Function of HIF-\{alpha\} Isoforms in Macrophages Are Essential for NO Homeostasis. Genes Dev. 2010, 24, 491-501. [CrossRef] [PubMed]

53. Wang, T.; Liu, H.; Lian, G.; Zhang, S.-Y.; Wang, X.; Jiang, C. HIF1 $\alpha$-Induced Glycolysis Metabolism Is Essential to the Activation of Inflammatory Macrophages. Mediators Inflamm. 2017, 2017, 9029327. [CrossRef] [PubMed]

54. Adam, J.; Hatipoglu, E.; O’Flaherty, L.; Ternette, N.; Sahgal, N.; Lockstone, H.; Baban, D.; Nye, E.; Stamp, G.W.; Wolhuter, K.; et al. Renal Cyst Formation in Fh1-Deficient Mice Is Independent of the Hif/Phd Pathway: Roles for Fumarate in KEAP1 Succination and Nrf2 Signaling. Cancer Cell 2011, 20, 524-537. [CrossRef] [PubMed]

55. Tannahill, G.M.; Curtis, A.M.; Adamik, J.; Palsson-McDermott, E.M.; McGettrick, A.F.; Goel, G.; Frezza, C.; Bernard, N.J.; Kelly, B.; Foley, N.H.; et al. Succinate Is an Inflammatory Signal That Induces IL-1 $\beta$ through HIF-1 $\alpha$. Nature 2013, 496, 238-242. [CrossRef] [PubMed]

56. Harber, K.J.; de Goede, K.E.; Verberk, S.G.S.; Meinster, E.; de Vries, H.E.; van Weeghel, M.; de Winther, M.P.J.; van den Bossche, J Succinate Is an Inflammation-Induced Immunoregulatory Metabolite in Macrophages. Metabolites 2020, 10, 372. [CrossRef]

57. Keiran, N.; Ceperuelo-Mallafré, V.; Calvo, E.; Hernández-Alvarez, M.I.; Ejarque, M.; Núñez-Roa, C.; Horrillo, D.; Maymó-Masip, E.; Rodríguez, M.M.; Fradera, R.; et al. SUCNR1 Controls an Anti-Inflammatory Program in Macrophages to Regulate the Metabolic Response to Obesity. Nat. Immunol. 2019, 20, 581-592. [CrossRef]

58. Ward, S. Heme Oxygenase-1: A Novel Anti-Inflammatory Mediator. Trends Immunol. 2002, 23, 430. [CrossRef]

59. Schmidt, E.A.; Fee, B.E.; Henry, S.C.; Nichols, A.G.; Shinohara, M.L.; Rathmell, J.C.; MacIver, N.J.; Coers, J.; Ilkayeva, O.R.; Koves, T.R.; et al. Metabolic Alterations Contribute to Enhanced Inflammatory Cytokine Production in Irgm1-Deficient Macrophages. J. Biol. Chem. 2017, 292, 4651-4662. [CrossRef]

60. Bafica, A.; Feng, C.G.; Santiago, H.C.; Aliberti, J.; Cheever, A.; Thomas, K.E.; Taylor, G.A.; Vogel, S.N.; Sher, A. The IFN-Inducible GTPase LRG47 (Irgm1) Negatively Regulates TLR4-Triggered Proinflammatory Cytokine Production and Prevents Endotoxemia. J. Immunol. 2007, 179, 5514. [CrossRef]

61. Kim, J.Y.; Ozato, K. The Sequestosome 1/P62 Attenuates Cytokine Gene Expression in Activated Macrophages by Inhibiting IFN Regulatory Factor 8 and TNF Receptor-Associated Factor 6/NF-KB Activity. J. Immunol. 2009, 182, 2131. [CrossRef] [PubMed]

62. Abrams, C.S.; Wu, H.; Zhao, W.; Belmonte, E.; White, D.; Brass, L.F. Pleckstrin Inhibits Phosphoinositide Hydrolysis Initiated by G-Protein-Coupled and Growth Factor Receptors. A ROLE FOR PLECKSTRIN'S PH DOMAINS *. J. Biol. Chem. 1995, 270, 14485-14492. [CrossRef] [PubMed]

63. Abrams, C.S.; Zhao, W.; Belmonte, E.; Brass, L.F. Protein Kinase C Regulates Pleckstrin by Phosphorylation of Sites Adjacent to the N-Terminal Pleckstrin Homology Domain (*). J. Biol. Chem. 1995, 270, 23317-23321. [CrossRef] [PubMed]

64. Dennis, E.A.; Deems, R.A.; Harkewicz, R.; Quehenberger, O.; Brown, H.A.; Milne, S.B.; Myers, D.S.; Glass, C.K.; Hardiman, G.; Reichart, D.; et al. A Mouse Macrophage Lipidome. J. Biol. Chem. 2010, 285, 39976-39985. [CrossRef] [PubMed]

65. Koganesawa, M.; Yamaguchi, M.; Samuchiwal, S.K.; Balestrieri, B. Lipid Profile of Activated Macrophages and Contribution of Group V Phospholipase A (2). Biomolecules 2020, 11, 25. [CrossRef] [PubMed]

66. Snider, S.A.; Margison, K.D.; Ghorbani, P.; LeBlond, N.D.; O’Dwyer, C.; Nunes, J.R.C.; Nguyen, T.; Xu, H.; Bennett, S.A.L.; Fullerton, M.D. Choline Transport Links Macrophage Phospholipid Metabolism and Inflammation. J. Biol. Chem. 2018, 293, 11600-11611. [CrossRef] 
67. Schober, C.; Schiller, J.; Pinker, F.; Hengstler, J.G.; Fuchs, B. Lysophosphatidylethanolamine Is-in Contrast to-CholineGenerated under in Vivo Conditions Exclusively by Phospholipase A2 but Not by Hypochlorous Acid. Bioorganic Chem. 2009, 37, 202-210. [CrossRef]

68. Park, K.S.; Lee, H.Y.; Lee, S.Y.; Kim, M.-K.; Kim, S.D.; Kim, J.M.; Yun, J.; Im, D.-S.; Bae, Y.-S. Lysophosphatidylethanolamine Stimulates Chemotactic Migration and Cellular Invasion in SK-OV3 Human Ovarian Cancer Cells: Involvement of Pertussis Toxin-Sensitive G-Protein Coupled Receptor. FEBS Lett. 2007, 581, 4411-4416. [CrossRef]

69. Martinon, F.; Mayor, A.; Tschopp, J. The Inflammasomes: Guardians of the Body. Annu. Rev. Immunol. 2009, 27, 229-265. [CrossRef]

70. Lamkanfi, M.; Kanneganti, T.-D.; Franchi, L.; Núñez, G. Caspase-1 Inflammasomes in Infection and Inflammation. J. Leukoc. Biol. 2007, 82, 220-225. [CrossRef]

71. Carneiro, A.B.; Iaciura, B.M.F.; Nohara, L.L.; Lopes, C.D.; Veas, E.M.C.; Mariano, V.S.; Bozza, P.T.; Lopes, U.G.; Atella, G.C.; Almeida, I.C.; et al. Lysophosphatidylcholine Triggers TLR2- and TLR4-Mediated Signaling Pathways but Counteracts LPSInduced NO Synthesis in Peritoneal Macrophages by Inhibiting NF-KB Translocation and MAPK/ERK Phosphorylation. PLoS ONE 2013, 8, e76233. [CrossRef] [PubMed]

72. Quan, H.; Hur, Y.-H.; Xin, C.; Kim, J.-M.; Choi, J.-I.; Kim, M.-Y.; Bae, H.-B. Stearoyl Lysophosphatidylcholine Enhances the Phagocytic Ability of Macrophages through the AMP-Activated Protein Kinase/P38 Mitogen Activated Protein Kinase Pathway. Int. Immunopharmacol. 2016, 39, 328-334. [CrossRef] [PubMed]

73. Jing, Q.; Xin, S.-M.; Zhang, W.-B.; Wang, P.; Qin, Y.-W.; Pei, G. Lysophosphatidylcholine Activates P38 and P42/44 MitogenActivated Protein Kinases in Monocytic THP-1 Cells, but Only P38 Activation Is Involved in Its Stimulated Chemotaxis. Circ. Res. 2000, 87, 52-59. [CrossRef] [PubMed]

74. Rodríguez-Prados, J.-C.; Través, P.G.; Cuenca, J.; Rico, D.; Aragonés, J.; Martín-Sanz, P.; Cascante, M.; Boscá, L. Substrate Fate in Activated Macrophages: A Comparison between Innate, Classic, and Alternative Activation. J. Immunol. 2010, 185, 605. [CrossRef]

75. Mills, E.L.; Kelly, B.; Logan, A.; Costa, A.S.H.; Varma, M.; Bryant, C.E.; Tourlomousis, P.; Däbritz, J.H.M.; Gottlieb, E.; Latorre, I.; et al. Succinate Dehydrogenase Supports Metabolic Repurposing of Mitochondria to Drive Inflammatory Macrophages. Cell 2016, 167, 457-470.e13. [CrossRef]

76. Nakayama, G.R.; Caton, M.C.; Nova, M.P.; Parandoosh, Z. Assessment of the Alamar Blue Assay for Cellular Growth and Viability in Vitro. J. Immunol. Methods 1997, 204, 205-208. [CrossRef]

77. Spickett, C.M.; Pitt, A.R.; Brown, A.J. Direct Observation of Lipid Hydroperoxides in Phospholipid Vesicles by Electrospray Mass Spectrometry. Free Radic. Biol. Med. 1998, 25, 613-620. [CrossRef]

78. Green, L.C.; Wagner, D.A.; Glogowski, J.; Skipper, P.L.; Wishnok, J.S.; Tannenbaum, S.R. Analysis of Nitrate, Nitrite, and [15N] Nitrate in Biological Fluids. Anal. Biochem. 1982, 126, 131-138. [CrossRef]

79. Amado, F.; Domingues, M.R.; Domingues, P.; Ferreira, R.; Vitorino, R. Análise de Proteínas—Guia do Laboratório, 1st ed.; 100Luz: Lisboa, Portugal, 2013; pp. 44-81.

80. Bligh, E.G.; Dyer, W.J. A rapid method of total lipid extraction and purification. Can. J. Biochem. Physiology 1959, 37, 911-917. [CrossRef]

81. Bartlett, E.M.; Lewis, D.H. Spectrophotometric Determination of Phosphate Esters in the Presence and Absence of Orthophosphate Anal. Biochem. 1970, 36, 159-167. [CrossRef]

82. Guerra, I.M.S.; Diogo, L.; Pinho, M.; Melo, T.; Domingues, P.; Domingues, M.R.; Moreira, A.S.P. Plasma Phospholipidomic Profile Differs between Children with Phenylketonuria and Healthy Children. J. Proteome Res. 2021, 20, 2651-2661. [CrossRef] [PubMed]

83. Mass Spectrometry-Data Independent Analysis (MS-DIAL). Available online: http://prime.psc.riken.jp/compms/msdial/main html (accessed on 5 July 2021).

84. Pluskal, T.; Castillo, S.; Villar-Briones, A.; Orešič, M. MZmine 2: Modular Framework for Processing, Visualizing, and Analyzing Mass Spectrometry-Based Molecular Profile Data. BMC Bioinformatics 2010, 11, 395. [CrossRef] [PubMed]

85. Yuan, M.; Breitkopf, S.B.; Yang, X.; Asara, J.M. A Positive/Negative Ion-Switching, Targeted Mass Spectrometry-Based Metabolomics Platform for Bodily Fluids, Cells, and Fresh and Fixed Tissue. Nat. Protoc. 2012, 7, 872-881. [CrossRef] [PubMed]

86. R: The R Project for Statistical Computing. Available online: https://www.r-project.org/ (accessed on 8 July 2021).

87. RStudio: Integrated Development Environment for R. Available online: http:/ / www.rstudio.com/ (accessed on 8 July 2021).

88. Karpievitch, Y.V.; Polpitiya, A.D.; Anderson, G.A.; Smith, R.D.; Dabney, A.R. Liquid Chromatography Mass Spectrometry-Based Proteomics: Biological and Technological Aspects. Ann. Appl. Stat. 2010, 4, 1797. [CrossRef] [PubMed]

89. Stacklies, W.; Redestig, H.; Scholz, M.; Walther, D.; Selbig, J. PcaMethods-A Bioconductor Package Providing PCA Methods for Incomplete Data. Bioinformatics 2007, 23, 1164-1167. [CrossRef]

90. Wickham, H. Ggplot2: Elegant Graphics for Data Analysis., 2nd ed.; Springer: New York, NY, USA, 2009.

91. pheatmap: Pretty Heatmaps. Available online: https://CRAN.R-project.org/package=pheatmap (accessed on 12 July 2021).

92. Xia, J.; Wishart, D.S. Using MetaboAnalyst 3.0 for Comprehensive Metabolomics Data Analysis. Curr. Protoc. Bioinforma. 2016, 55, 14.10.1-14.10.91. [CrossRef] 